\title{
Cell Death Triggered by a Putative Amphipathic Helix of Radish mosaic virus Helicase Protein Is Tightly Correlated With Host Membrane Modification
}

\author{
Masayoshi Hashimoto, ${ }^{1}$ Ken Komatsu, ${ }^{2}$ Ryo Iwai, ${ }^{1}$ Takuya Keima, ${ }^{1}$ Kensaku Maejima, ${ }^{1}$ \\ Takuya Shiraishi, ${ }^{1}$ Kazuya Ishikawa, ${ }^{1}$ Tetsuya Yoshida, ${ }^{1}$ Yugo Kitazawa, ${ }^{1}$ Yukari Okano, ${ }^{1}$ \\ Yasuyuki Yamaji, ${ }^{1}$ and Shigetou Namba ${ }^{1}$ \\ ${ }^{1}$ Laboratory of Plant Pathology, Department of Agricultural and Environmental Biology, Graduate School of Agricultural and Life \\ Sciences, The University of Tokyo, 1-1-1 Yayoi, Bunkyo-ku, Tokyo 113-8657, Japan; ' 2aboratory of Plant Pathology, Tokyo \\ University of Agriculture and Technology (TUAT), 3-5-8 Saiwaicho, Fuchu, Tokyo 183-8509, Japan
}

Submitted 6 January 2015. Accepted 23 January 2015.

\begin{abstract}
Systemic necrosis is one of the most severe symptoms caused by plant RNA viruses. Recently, systemic necrosis has been suggested to have similar features to a defense response referred to as the hypersensitive response (HR), a form of programmed cell death. In virus-infected plant cells, host intracellular membrane structures are changed dramatically for more efficient viral replication. However, little is known about whether this replication-associated membrane modification is the cause of the symptoms. In this study, we identified an amino-terminal amphipathic helix of the helicase encoded by Radish mosaic virus (RaMV) (genus Comovirus) as an elicitor of cell death in RaMV-infected plants. Cell death caused by the amphipathic helix had features similar to HR, such as SGT1-dependence. Mutational analyses and inhibitor assays using cerulenin demonstrated that the amphipathic helix-induced cell death was tightly correlated with dramatic alterations in endoplasmic reticulum (ER) membrane structures. Furthermore, the cell death-inducing activity of the amphipathic helix was conserved in Cowpea mosaic virus (genus Comovirus) and Tobacco ringspot virus (genus Nepovirus), both of which are classified in the family Secoviridae. Together, these results indicate that ER membrane modification associated with viral intracellular replication may be recognized to prime defense responses against plant viruses.
\end{abstract}

One of the most important plant defense responses against viruses is resistance $(\mathrm{R})$-mediated disease resistance. This is often associated with the hypersensitive response (HR), a form of programmed cell death (PCD) that confines pathogens to the initial infection site. Systemic HR (SHR) is regarded as an inefficient defense response, which can also be induced during $\mathrm{R}$ protein-plant virus interactions (Dinesh-Kumar et al. 2000; Moffett 2009; Sekine et al. 2006; Seo et al. 2006). During SHR, R-mediated defense machinery does not prevent the spread of a virus, resulting in eventual induction of $\mathrm{HR}$ responses in virus-infected systemic leaves. Several lines of evidence suggest that systemic necrosis, one of the most severe symptoms of

Corresponding author: S. Namba; E-mail: anamba@mail.ecc.u-tokyo.ac.jp

*The $\boldsymbol{e}$-Xtra logo stands for "electronic extra" and indicates that four supplementary figures and two supplementary tables are published online.

(c) 2015 The American Phytopathological Society plant virus infections, is equivalent to SHR, at least in some plant-virus interactions (Komatsu et al. 2010; Mandadi and Scholthof 2013; Xu and Roossinck 2000; Xu et al. 2003).

All studied positive-strand RNA viruses replicate their genomic RNA using host intracellular membranous structures (Schwartz et al. 2002). Positive-strand RNA viruses induce membrane modifications and the formation of vesicle structures to produce "virus replication factories" for their efficient replication in infected cells (den Boon and Ahlquist 2010; Diaz et al. 2010; Miller and Krijnse-Locker 2008). Such membrane modification is attributable to the action of membrane-targeted viral proteins such as replicases. When expressed alone, these viral proteins can induce membrane modifications similar to those observed in virus-infected cells. Targeting of these viral proteins to membranes is usually mediated by their transmembrane domains and amphipathic helices (den Boon et al. 2001; Laliberté and Sanfaçon 2010; Miller and Krijnse-Locker 2008). Previous studies on animal and plant viral replicases indicated that amphipathic helices, which have one polar side and one hydrophobic side, are responsible for vesicle formation in virus-infected cells and are required for virus replication (Drin and Antonny 2010; Gouttenoire et al. 2010; Horner et al. 2012; Liu et al. 2009). Vesicle formation by amphipathic helices is controlled by association of the helix structure with one of the two leaflets of the membrane, thereby creating asymmetry and membrane bending (Miller and Krijnse-Locker 2008). In plant cells in which host membranes are modified by virus replication, membrane physiological states such as membrane synthesis and trafficking are changed dramatically. We suggest that these changes in host membrane physiology in virus-infected cells contribute to symptom expression, including necrosis. However, the relationship between replicationassociated membrane modification and symptom expression remains unclear.

The comoviruses are a group of plant viruses in the picornavirus superfamily that have a genomic organization similar to that of animal picornaviruses (Sanfaçon 2012; Sanfaçon et al. 2009). RNA-1 in members of genus Comovirus, such as Cowpea mosaic virus (CPMV) and Bean pod mottle virus (BPMV), encodes five mature proteins required for replication, including the helicase (Hel) protein. Upon infection with CPMV, the CPMV-encoded proteinase cofactor (CoPro) and Hel proteins are responsible for the proliferation of the endoplasmic reticulum (ER) membrane, which is essential for viral replication (Carette et al. 2002). Additionally, such membrane 
modification requires new membrane synthesis, which is inhibited by cerulenin treatment (Carrette et al. 2000). Overexpression of the Hel protein, encoded by CPMV and BPMV, results in cell death (Carette et al. 2002; Gu and Ghabrial 2005). However, the mechanism of cell death induction by the Hel protein remains unclear, especially in terms of the relationship with the ER membrane modification activity.

Radish mosaic virus (RaMV) is a member of genus Comovirus and infects most cruciferous plants, causing severe symptoms including enations, mosaic, and ringspots associated with necrosis (Komatsu et al. 2013). In this study, we identified the RaMV Hel protein as an elicitor of cell death in RaMV-infected Nicotiana benthamiana plants. The N-terminal region of the Hel protein (nHel), including an amphipathic helix, was responsible for the induction of cell death, which had several features similar to HR. Our results indicate that the ER membrane modification by the amphipathic helix of nHel was essential for virus replication and may contribute to the induction of cell death. Furthermore, we showed that cell death can also be induced by amphipathic helices from other viruses related to RaMV. Based on these findings, the possible mechanism of amphipathic helix-induced cell death is discussed.

\section{RESULTS}

\section{Necrosis in RaMV-infected $N$. benthamiana} has characteristics of HR.

RaMV can infect $N$. benthamiana systematically and produce severe symptoms with necrotic responses in shoot tips and surrounding leaves at 13 days postinfiltration (dpi) (Fig. 1A). We investigated whether the necrotic reactions induced by RaMV were related to plant defense responses. In previous studies, we showed that necrotic symptoms in $N$. benthamiana caused by Plantago asiatica mosaic virus (PlAMV) were consistent with several characteristics of HR (Komatsu et al. 2010).
Similar to responses to PlAMV infection, transcript levels of the defense-related genes PR-la, Hinl, and Hsr203j were found, using real-time reverse transcription polymerase chain reaction (RT-PCR) analysis, to be elevated in RaMV-inoculated $N$. benthamiana leaves at $5 \mathrm{dpi}$ (Fig. 1B). PR-la and Hinl transcript levels also increased significantly in upper uninoculated leaves (Supplementary Fig. S1).

To determine whether infection of tissues by RaMV is accompanied by nuclear DNA fragmentation that occurs during apoptosis-like PCD, we performed terminal deoxyribonucleotidyl transferase-mediated dUTP nick-end labeling (TUNEL) assays on shoot tips, in which necrotic responses would be induced. Green fluorescence representing nuclear DNA fragmentation was detected in positive control sections from healthy tissues treated with DNase I (Fig. 1C, left panel) but not in negative control sections from untreated tissues (Fig. 1C, center panel). In sections from RaMV-infected tissues, green fluorescence was detected throughout the tissues (Fig. 1C, right panel). These results suggest that the necrotic responses induced in RaMV-infected plants have several characteristics in common with the HR.

\section{Hel protein is a necrotic response elicitor in RaMV-infected $N$. benthamiana.}

To identify the viral elicitor that induces the necrotic response upon RaMV infection, we attempted to express each protein encoded by the RaMV genome (Fig. 2A) in N. benthamiana leaves using a Potato virus $X$ (PVX)-based expression system (Chapman et al. 1992). We found that only PVX:Hel induced cell death in the infiltrated area at $4 \mathrm{dpi}$, but neither other PVX constructs nor the PVX control vector did so (Supplementary Fig. S2). Consistent with these results based on the PVX vector system, cell death was also observed when yellow fluorescent protein (YFP)-tagged Hel protein was transiently expressed with agroinfiltration at 5 dpi (Fig. 2B and C) (Earley et al. 2006). Staining with $3,3^{\prime}$-diaminobenzidine (DAB) was performed to
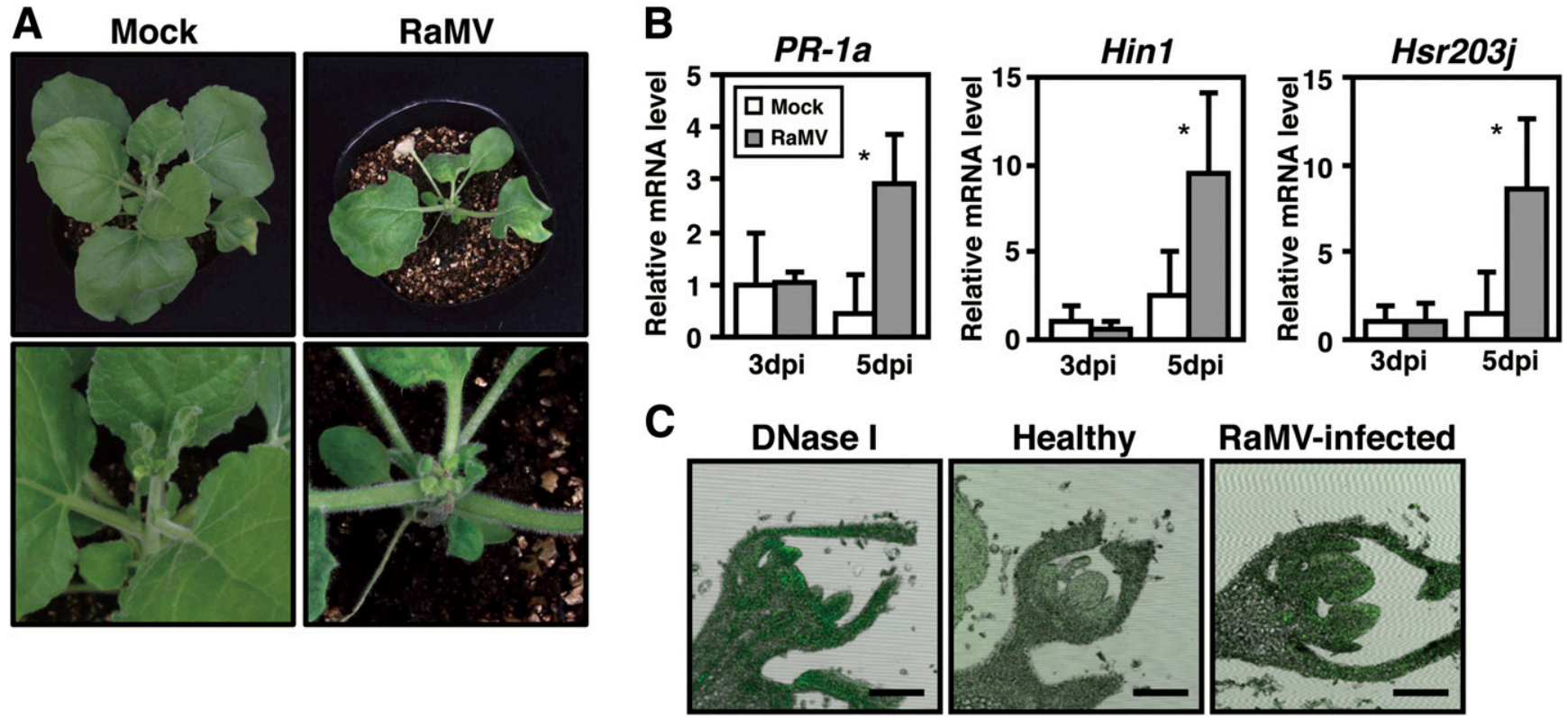

Fig. 1. Characterization of necrotic symptoms induced by Radish mosaic virus (RaMV)-J infection in Nicotiana benthamiana. A, Symptoms in RaMV-infected (right panels) or mock-inoculated plants. A pBI121 binary vector expressing $\beta$-glucuronidase was used as a mock control. Photos were taken at 13 days postinfiltration (dpi). The lower panel shows a close-up view of the shoot tip and its surrounding leaves shown in each upper panel. Experiments were repeated at least three times with similar results. B, Expression analysis of defense-related genes $P R$ - $1 a$, Hin 1, and $H s r 203 j$ in RaMV-infiltrated leaves using real-time reverse transcription-polymerase chain reaction analysis. $N$. benthamiana leaves were infiltrated with the pBI121 binary vector (mock) or RaMV at an optical density at $600 \mathrm{~nm}$ of 0.05 . Values presented are relative to those of mock-infiltrated areas at 3 dpi. Data shown represent means \pm standard deviation of five independent plants. Asterisks indicate statistically significant differences from mock-infiltrated areas at each time point $(P<0.05)$. C, In situ detection of nuclear DNA fragmentation in the RaMV-infected plants by transferase-mediated dUTP nick-end labeling assay. Sections were prepared from RaMV-infected (right panel) or healthy (left and center panels) $N$. benthamiana plants at $10 \mathrm{dpi}$. The left panel shows the positive control using the sections derived from healthy plants treated with DNase I. The green signals indicate nuclear DNA fragmentation. Bars represent $200 \mu \mathrm{m}$. 
detect hydrogen peroxide $\left(\mathrm{H}_{2} \mathrm{O}_{2}\right)$ production, one of the hallmarks of HR. Reddish-brown staining in the YFP:Hel-infiltrated area, but not in the Myc:green fluorescent protein (GFP)infiltrated area (Fig. 2B, lower panels), indicated that YFP:Helinduced cell death was associated with $\mathrm{H}_{2} \mathrm{O}_{2}$ production.

To examine the expression and distribution of Hel in meristems of RaMV-infected plants, in which cell death would be induced, we performed immunohistochemical analysis using anti-Hel antibody. RaMV- and mock-inoculated shoot meristematic tissues were collected at $10 \mathrm{dpi}$. When anti-LCP (large coat protein) antibody was used, a strong signal was detected throughout the shoot tips of RaMV-infected plants (Fig. 2D, left panel), indicating that RaMV invaded $N$. benthamiana plants systemically by $10 \mathrm{dpi}$, as reported previously (Komatsu et al. 2013). Similarly, a Hel protein signal was detected throughout the shoot tips of RaMV-infected plants (Fig. 2D, center panel). No Hel or LCP signals were identified in healthy plants (Fig. $2 \mathrm{D}$, right panel and data not shown), confirming the expression of Hel in the shoot tips of RaMV-infected plants prior to the induction of cell death. These signals are likely to coincide with PCD-induced tissues shown by the TUNEL assay (Fig. 1C). Note that these specific signals using anti-Hel antibody would not necessarily be consistent with the localization of the mature Hel protein because comoviruses, including RaMV, produce mature proteins and cleavage intermediates by viral protease-mediated polyprotein processing. However, our result that overexpression of the Hel-Vpg (viral protein genome-linked) fusion protein also causes cell death (S. Namba and M. Hashimoto, unpublished data) suggests that other intermediates containing Hel could have the potential to induce cell death in RaMV-infected tissues. Collectively, these results suggest that the RaMV-Hel protein may act as an elicitor of HR-like cell death in RaMV-infected $N$. benthamiana that is most prominent in the shoot tips (Fig. 1A).

\section{The amino-terminal region of RaMV-Hel is responsible for inducing cell death.}

We analyzed the functional domain structure of Hel to identify the region responsible for inducing cell death. Analysis using TMPred software predicted two transmembrane helices at amino acid (aa) positions 5 to 25 and 551 to 590 of $\mathrm{Hel}$. AmphipaSeek software and the Consensus secondary structure prediction method software predicted an amphipathic $\alpha$-helix at aa 36 to 60 in the $\mathrm{N}$-terminal region of Hel.

Based on these domain structure predictions, we divided Hel into three regions: an $\mathrm{N}$-terminal region containing one transmembrane helix and one amphipathic helix (nHel), a middle region containing no hydrophobic stretches (mHel), and a $\mathrm{C}$-terminal region containing one transmembrane helix (cHel) (Fig. 3A). We expressed N-terminal fusion proteins of each region transiently with YFP (Fig. 3B). At 3 dpi, cell death was clearly observed in areas infiltrated with YFP:nHel but not with YFP:mHel or YFP:cHel (Fig. 3C, upper panels). To exclude any potential artifacts caused by the YFP tag, we also created N-terminal Myc-tagged constructs of each region. At 3 dpi, transient overexpression of Myc:nHel but not of Myc:mHel or Myc:cHel induced cell death (data not shown; Supplementary Fig. S3), suggesting that cell death induced by YFP:nHel and Myc:nHel reflected the native function of the nHel region in cell death.

To investigate whether the cell death elicited by $\mathrm{nHel}$ retained the characteristics of defense responses similar to those induced in RaMV-infected tissues, we performed several assays. DAB staining showed that cell death in the areas infiltrated with YFP:nHel was accompanied by $\mathrm{H}_{2} \mathrm{O}_{2}$ production, whereas no signal was detected in the areas infiltrated with YFP:mHel or YFP:cHel (Fig. 3C, lower panels). Real-time RT-PCR analysis to quantify the expression of defense-related genes showed that Hinl and Hsr203j mRNA levels in the areas infiltrated with YFP:nHel were significantly higher than those in the areas infiltrated with YFP:mHel or YFP:cHel at 2 dpi (Fig. 3D). In contrast, $P R-1 a$ expression in the YFP:nHelinfiltrated areas was not significantly different from those infiltrated with other fusion proteins. This is probably because agroinfiltration itself induces a high level of $P R-1 a$ expression in infiltrated areas of $N$. benthamiana (Pruss et al. 2008). SGT1 functions as an essential component of R-mediated disease resistance to bacterial and eukaryotic pathogens as well as viruses (Azevedo et al. 2002; Peart et al. 2002). We previously showed that SGT1 was also involved in systemic necrosis caused by PlAMV (Komatsu et al. 2010). To test whether SGT1 is required for YFP:nHel-triggered cell death, we used the Tobacco rattle virus-based virus-induced gene silencing method (Ratcliff et al. 2001). YFP:nHel was transiently overexpressed in the upper uninoculated leaves of NbSGT1-silenced or nonsilenced plants. We found that cell death induced by YFP:nHel was

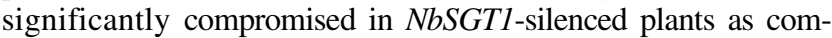
pared with nonsilenced control plants (Fig. 3E). Taken together, these results indicate that the $\mathrm{nHel}$ region, containing one putative

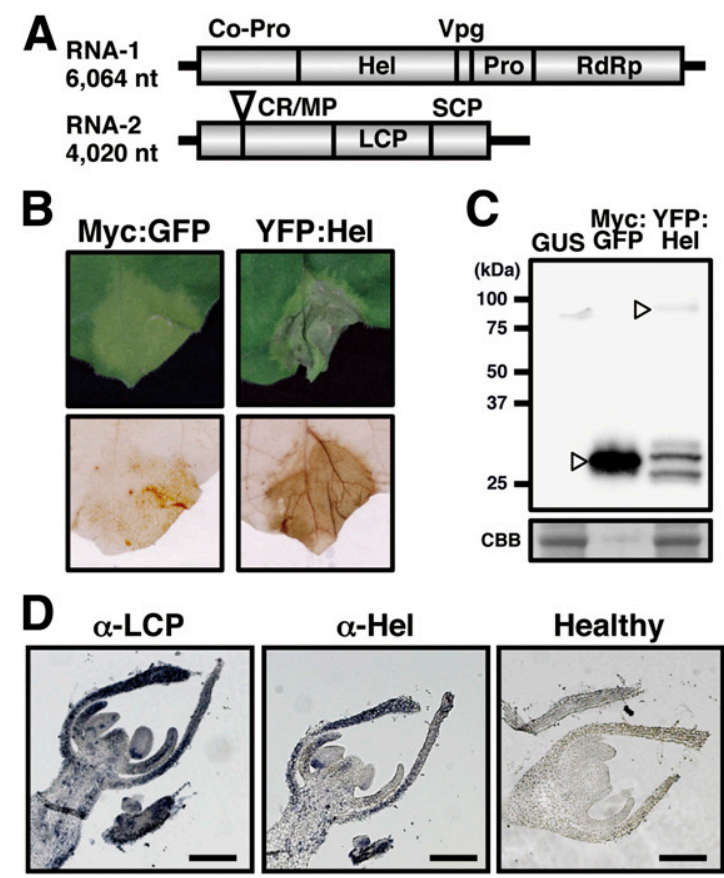

Fig. 2. Involvement of the Radish mosaic virus (RaMV)-encoded Hel protein in the necrotic symptoms induced by RaMV infection. A, Genomic organization of the RaMV genome. The RaMV genome consists of two positive single-stranded RNA molecules (RNA-1 and RNA-2). Open reading frames and untranslated regions in the RNA molecules are indicated by open boxes and horizontal lines, respectively. Co-Pro $=$ cofactor for proteinase, $\mathrm{VPg}=$ viral protein genome-linked, $\mathrm{CR}=$ cofactor for replication, $\mathrm{MP}=$ movement protein, $\mathrm{LCP}=$ large coat protein, $\mathrm{SCP}=$ small coat protein. The second start codon of RNA-2 is indicated by an arrowhead. B, Symptoms of leaves infiltrated with Myc-tagged green fluorescent protein (Myc:GFP; upper left panel) or yellow fluorescent protein-tagged RaMV-Hel (YFP:Hel; upper right panel) at an optical density at $600 \mathrm{~nm}$ of 0.5 and $3,3^{\prime}$-diaminobenzidine staining of the same leaves (lower panels). Photos were taken at 6 days postinfiltration (dpi). C, Western blot analysis of YFP-tagged Hel protein using a monoclonal anti-GFP (green fluorescent protein) antibody (upper panel). Arrowheads indicate specific signals of YFP:Hel protein or Myc:GFP. Myc:GFP was used as a control. Migration of molecular mass standards is indicated on the left. A Coomassie Brilliant Blue-stained gel of identical protein samples is shown as a loading control (lower panel). D, Distribution of large coat proteins (LCP) and Hel proteins in the shoot tips of RaMV-infected plants at 10 dpi. Blue signals were detected using anti-LCP (left panel) or anti-Hel antibody (center panel). The right panel shows a negative control derived from samples of healthy plants treated with the anti-Hel antibody. Bars represent $200 \mu \mathrm{m}$. 
transmembrane domain and an amphipathic helix, is responsible for cell death having several features in common with HR.

The N-terminal region of RaMV-Hel induces

the formation of perinuclear aggregates

and dramatic rearrangement of the ER network.

Based on the finding that the N-terminal region of Hel containing a putative transmembrane helix and an amphipathic

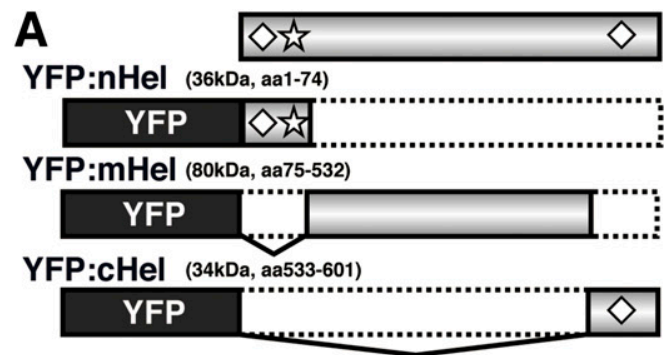

B

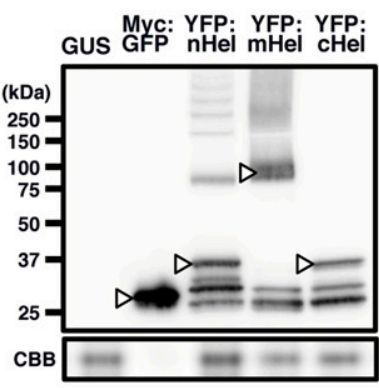

C

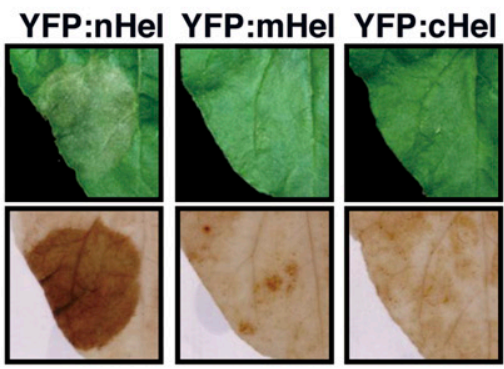

D
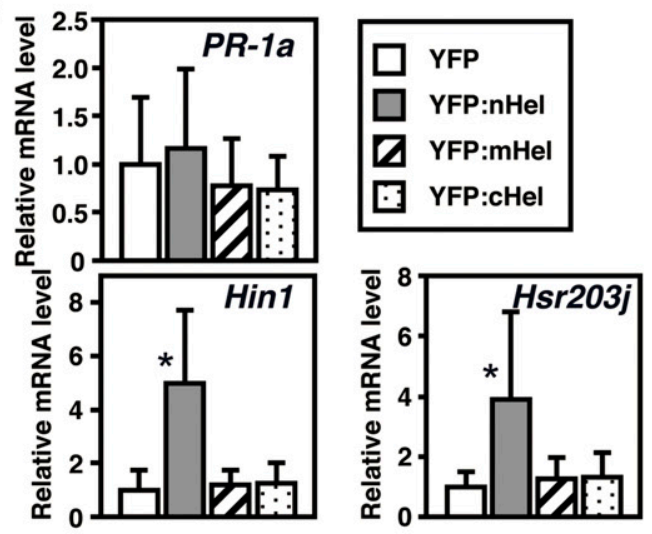

E

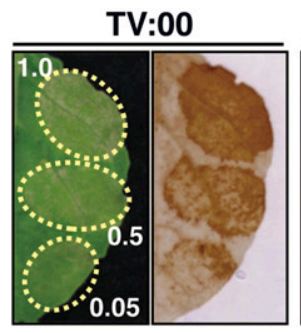

TV:NbSGT1

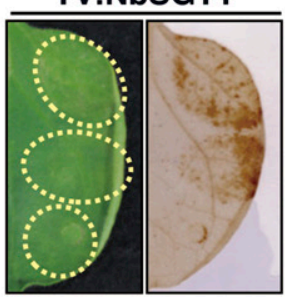

helix is responsible for cell death, we considered whether intracellular localization of Hel might play an important role in the induction of cell death. To investigate the localization of Hel using confocal laser scanning microscopy (CLSM), we coinfiltrated YFP:Hel into $N$. benthamiana leaves with ERtargeted cyan fluorescent protein (ER-CFP) (Nelson et al. 2007) to compare their localization. At $3 \mathrm{dpi}$, the fluorescence of YFP: Hel coincided with that of ER-CFP (Fig. 4A, row 1). YFP:Hel formed aggregates that colocalized with the ER membrane in the perinuclear area (Fig. 4A, arrows in row 2). YFP:Hel also induced granular structures resulting in morphological changes in the ER network at the peripheral parts of cells (Fig. 4A, arrowheads in row 3 ). The cell death-inducing activity of the Hel protein was retained with the N-terminal YFP fusion, suggesting that the YFP fusion does not influence the cell death-inducing activity of the native Hel protein (Fig. 2B). C-terminal YFP fusion to full-length Hel protein produced no fluorescence (data not shown).

We next investigated the subcellular localization of each region of $\mathrm{Hel}$ (nHel, mHel, and cHel) using CLSM. We coexpressed each YFP-tagged construct with ER-CFP in $N$. benthamiana leaves. At $3 \mathrm{dpi}$, the fluorescence of YFP:cHel coincided with the ER-CFP fluorescence and formed giant aggregates in the cytoplasm (Fig. 4B, arrows in row 1). In the peripheral parts of the cells, YFP:cHel colocalized with the cortical ER network and occasionally formed granular structures that were closely associated with the ER network (Fig. 4B, arrowheads in row 2). YFP:mHel fluorescence was localized to the cytoplasm and nucleus, similar to the localization of transiently-expressed free GFP (Fig. 4B, row 3). In row 3 of Figure 4B, YFP:mHel fluorescence seemed to partially coincide with that of ER-CFP, but it appeared dispersed in the cytoplasm at high magnification (Fig. 4B, row 4), confirming that YFP:mHel did not colocalize with the ER membrane. No fluorescence of YFP:nHel was observed at 3 dpi, probably due to rapid induction of cell death in the YFP:nHel-infiltrated areas (data not shown). Thus, we tried to examine YFP:nHel fluorescence at earlier time points. At 2 dpi, the YFP:nHel fluorescence colocalized with the ER-CFP fluorescence (Fig. $4 \mathrm{~B}$, row 5) and formed aggregates in the perinucleus (Fig. 4B, arrows in rows 5 and 6 ). The ER network structure was also dramatically changed, and many granular structures were formed

Fig. 3. Characterization of cell death induced by the N-terminal region of the Hel protein (nHel) of the Radish mosaic virus (RaMV)-Hel protein. A, Schematic representation of truncated proteins derived from the Hel protein tagged with yellow fluorescent protein (YFP). Black and gray boxes represent YFP and partial fragments of the Hel protein, respectively. The putative amphipathic helix and transmembrane helices are represented by a star and diamonds, respectively. B, Western blot analysis of YFP-tagged Hel fragments using a monoclonal anti-GFP (green fluorescent protein) antibody. Arrowheads indicate specific signals of YFP-tagged proteins or Myc:GFP. C, Local symptoms induced by overexpression of YFP:nHel (upper left panel), YFP:mHel (center panel), or YFP:cHel (right panel) at an optical density at $600 \mathrm{~nm}\left(\mathrm{OD}_{600}\right)$ of 0.5 and $3,3^{\prime}$-diaminobenzidine (DAB) staining of the same leaves (lower panels). Photos were taken at 3 days postinfiltration (dpi). D, Expression analysis of defense-related genes in areas infiltrated with YFP-tagged Hel fragments at an $\mathrm{OD}_{600}$ of 0.05. Total RNAs were extracted from infiltrated areas at 2 dpi. The values are represented relative to those of YFP-infiltrated areas. Data shown represent means \pm standard deviation of seven independent plants. Asterisks indicate statistically significant differences from YFPinfiltrated areas $(P<0.05)$. E, Symptoms of areas infiltrated with YFP:nHel in NbSGT1-silenced (TV:NbSGT1, right two panels) or nonsilenced plants (TV:00, left two panels) and DAB staining of the same leaves (respective right panels). YFP:nHel was agroinfiltrated into yellowcircled areas at $\mathrm{OD}_{600}$ values of 1.0, 0.5, and 0.05, as shown in the left panel. Photos were taken at $4 \mathrm{dpi}$. 
A

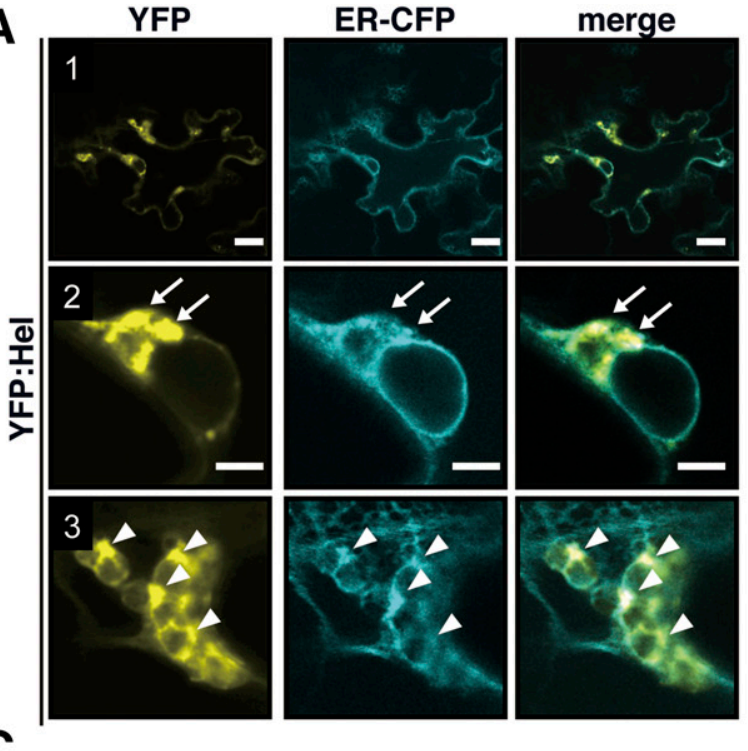

C
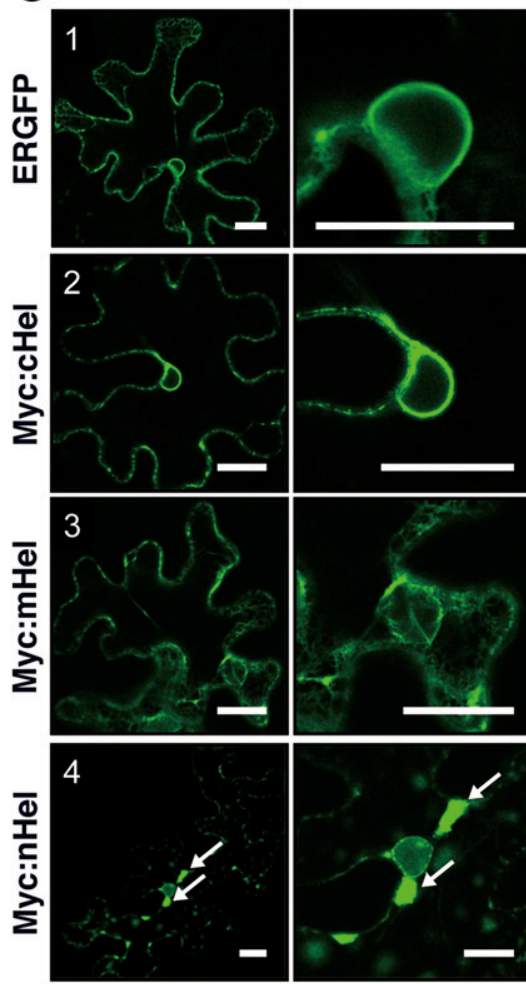

D

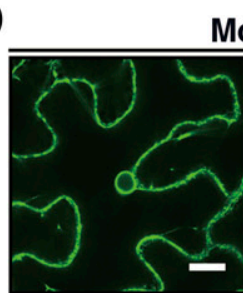

Mock

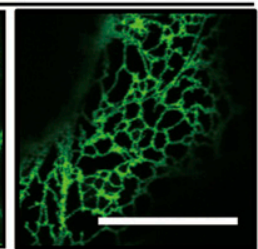

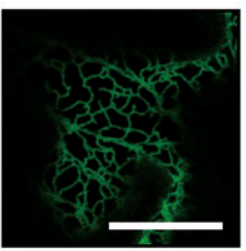

B
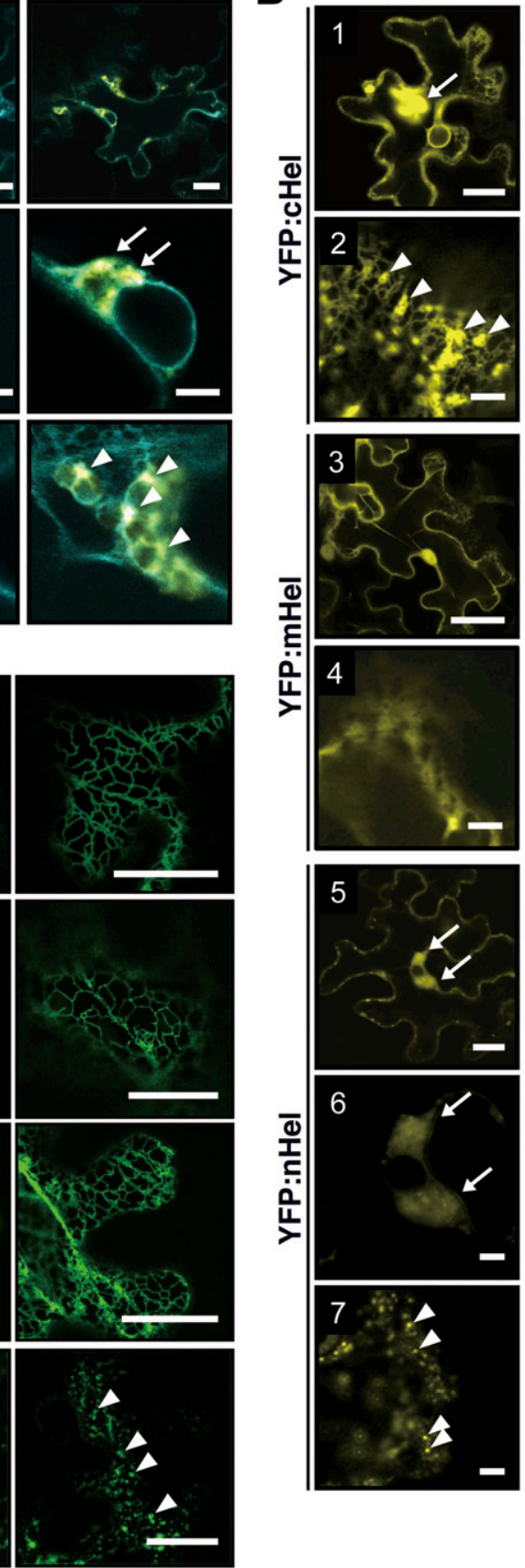
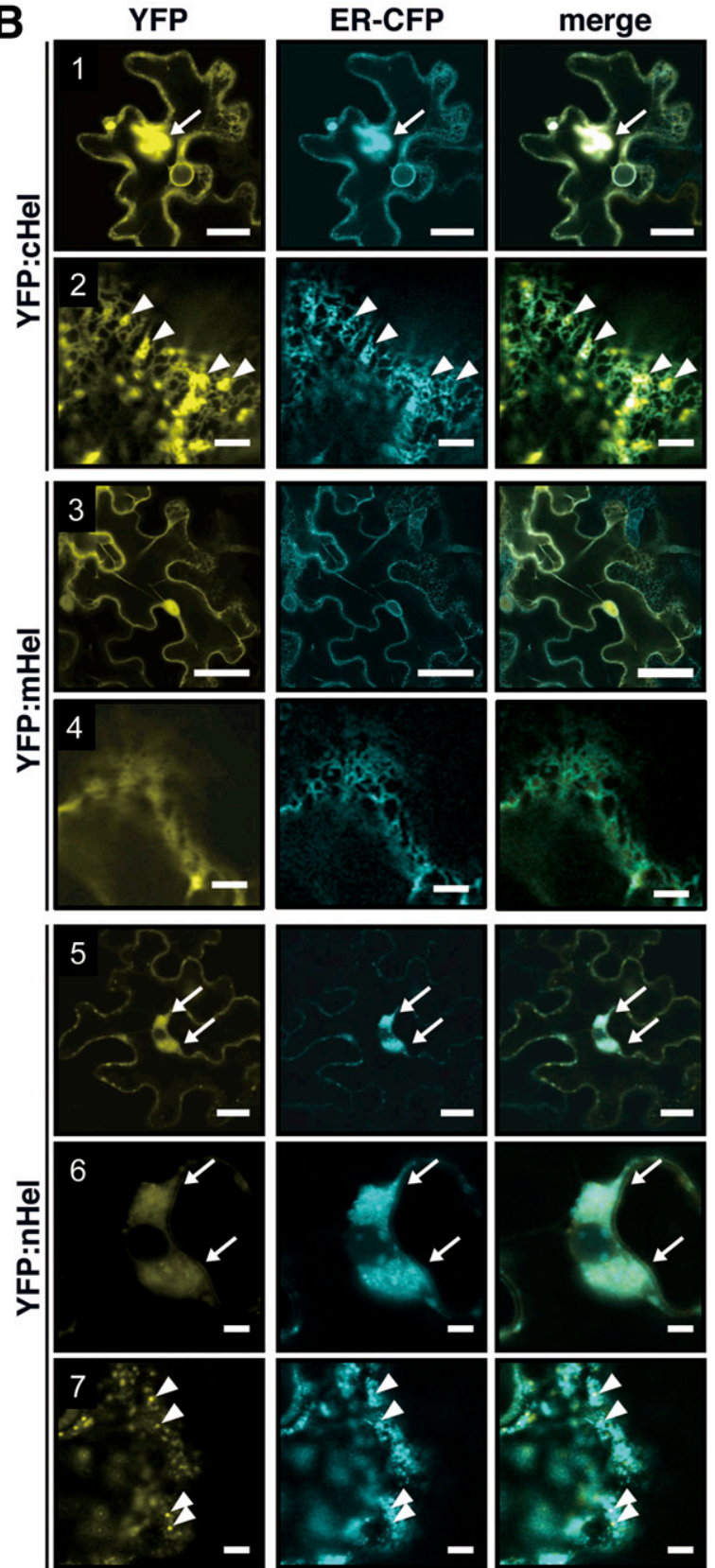

Fig. 4. Identification of the N-terminal region of the Hel protein (nHel) of Hel required for endoplasmic reticulum (ER) modification in Radish mosaic virus (RaMV)infected cells. A, Subcellular localization analysis of yellow fluorescent protein (YFP):Hel. YFP:Hel and ER-CFP (cyan fluorescent protein) constructs were agroinfiltrated at an optical density at $600 \mathrm{~nm}\left(\mathrm{OD}_{600}\right)$ of 0.1 . Panels 2 or 3 present close-up views of the perinuclear or the peripheral parts, respectively, of cells. Bars represent $20 \mu \mathrm{m}$ in row 1 and $5 \mu \mathrm{m}$ in rows 2 and 3. Except in B (row 1), arrows and arrowheads indicate perinuclear aggregates and granular structures, respectively. B, Subcellular localization analysis of YFP-tagged Hel fragments. Panels 6 or 2, 4, and 7 present close-up views of the perinuclear or peripheral parts of cells, respectively. Bars represent $20 \mu \mathrm{m}$ in rows 1,3 , and 5, and $5 \mu \mathrm{m}$ in rows 2, 4, 6, and 7. In row 1, arrows indicate giant aggregates in the cytoplasm. C, Subcellular localization analysis of ER-GFP in cells expressing Myc-tagged Hel fragments. ER-GFP was used as a control (row 1). Myc:cHel (row 2) or Myc:mHel (row 3) was coexpressed at an $\mathrm{OD}_{600}$ of 0.5 together with ER-GFP at an $\mathrm{OD}_{600}$ of 0.00025 . Photos were taken at 2 days postinfiltration (dpi). Row 4 shows the same leaves infiltrated with Myc:nHel, at 1 day after ER-GFP was expressed. Photos were taken after an additional $24 \mathrm{~h}$. Bars in each panel represent $20 \mu$ m. D, Subcellular localization analysis of ER-GFP in RaMV-infected plants. Nicotiana benthamiana plants of line 16c were infiltrated with $\beta$-glucuronidase (mock; left two panels) or RaMV (virusinfected; right two panels). GFP fluorescence of ER-GFP was observed in the noninfiltrated upper leaves at 7 dpi. Bars in each panel represent $20 \mu \mathrm{m}$. 
in the peripheral parts of cells (Fig. 4B, arrowheads in row 7). $\mathrm{C}$-terminal fusions of YFP to $\mathrm{nHel}$ and $\mathrm{cHel}$ proteins produced no fluorescence, perhaps due to misfolding of the fusion proteins caused by C-terminal fusion with YFP, similar to C-terminal fusion of YFP to full-length Hel protein (data not shown).

We examined whether ER membrane-derived structures were also formed by the overexpression of Myc-tagged Hel regions. Following coinfiltration of Myc:nHel, Myc:mHel, or Myc:cHel along with ER-targeted GFP (ER-GFP), we observed GFP fluorescence of ER-GFP at $2 \mathrm{dpi}$. Although reticular structures of the ER remained intact in the Myc:cHel- or Myc:mHel-coinfiltrated areas (Fig. 4C, rows 2 and 3, respectively) compared with the areas infiltrated with ER-GFP alone (Fig. 4C, row 1), no fluorescence was observed in the Myc:nHel-coinfiltrated areas, possibly due to collapse of the cells caused by cell death at $2 \mathrm{dpi}$ (data not shown). To eliminate the influence of cell death, we infiltrated Myc:nHel at $24 \mathrm{~h}$ after the infiltration of ER-GFP and examined GFP fluorescence of ER-GFP after an additional $24 \mathrm{~h}$. Perinuclear aggregates of the ER membrane were observed (Fig. 4C, row 4, arrows in the left and center panels), and many granular structures and drastic rearrangement of the ER network structure were induced in the peripheral parts of cells (Fig. 4C, row 4, arrowheads in the right panel).

To confirm whether the ER membrane structures were influenced in a similar manner by viral infection, we inoculated RaMV onto leaves of transgenic $N$. benthamiana plants expressing ER-GFP (line 16c) (Ruiz et al. 1998) and examined morphological changes in the ER membrane. This transgenic $N$. benthamiana line has been widely used as an experimental material to examine ER membrane structures in plants (Carette et al. 2002; Goodin et al. 2005; Turner et al. 2004). At 7 dpi, perinuclear membrane aggregates and granular structures associated with disruption of the ER network were observed in the peripheral parts of cells in the RaMV-infected plants (Fig. 4D, right two panels), similar to those observed in the Myc: nHel-infiltrated areas (Fig. 4C), but not in the mock-inoculated plants (Fig. 4D, left two panels).

These results demonstrate that both the $\mathrm{N}$ - and the $\mathrm{C}$-terminal regions of the Hel protein have the potential to recruit the Hel protein to the ER membrane. Moreover, the nHel region contributed to the ER membrane modification characterized by perinuclear aggregates and the rearrangement of the ER network structures observed in both YFP:Hel-expressing and RaMV-infected tissues. Similar perinuclear aggregates and ER granular structures are observed in CPMV-infected plants, and have been implicated in viral replication (Carette et al. 2000). Thus, nHel-induced ER membrane modification may be involved in RaMV replication.

\section{The N-terminal amphipathic helix of Hel is responsible for cell death and ER membrane modification.}

The nHel region, which showed cell death-inducing activity, was predicted to possess a putative transmembrane helix (aa 5 to 25 ) and an amphipathic helix (aa 36 to 60). To determine the minimal $\mathrm{nHel}$ region sequence required for ER membrane association and the induction of cell death, we constructed several deletion mutants of the nHel region (N-terminal 74 aa of RaMVHel) (Fig. 5A). The N-terminus of each mutant was fused to YFP and was transiently overexpressed in $N$. benthamiana leaves. At $5 \mathrm{dpi}$, overexpression of YFP:21-74 lacking the N-terminal 20 aa of RaMV-Hel led to cell death, but overexpression of YFP:28-74 lacking the N-terminal 27 aa did not. However, overexpression of only the N-terminal 27 aa (YFP:1-27) of the nHel region did not induce cell death. In contrast, YFP:1-60 and YFP:1-50, lacking the C-terminal 14 and 24 aa, respectively, caused cell death. These results indicated that the minimum sequence of the nHel region responsible for induction of cell death mapped to its central portion, from aa 21 to aa 50 . This sequence was predicted to contain most of the putative amphipathic helix and only five amino acids of the transmembrane helix.

To investigate the relationship between the cell deathinducing activity and the subcellular localization of the nHel region, we focused on two of the mutants, YFP:21-74 and YFP: 28-74, which possess different cell death-inducing activities. We explored their precise subcellular distribution, using CLSM at 40 and $48 \mathrm{~h}$ postinoculation (hpi) (Fig. 5B and C, respectively). At $40 \mathrm{hpi}$, in the perinuclear region of the cells, perinuclear aggregates derived from the ER membrane were observed in the YFP:21-74-infiltrated areas (Fig. 5B, row 1), but no aggregates were detected in the YFP:28-74-expressing areas (Fig. 5B, row 3). In contrast, in the peripheral areas of the cells expressing YFP:21-74 or YFP:28-74, granular structures were observed in close proximity to the ER network (Fig. 5B, arrowheads in rows 2 and 4). At 48 hpi, the ER network structure was largely destroyed in the area infiltrated with YFP:21-74 and granular structures still colocalized with the ER-CFP fluorescence (Fig. 5C, arrowheads in row 1). In contrast, although granular structures were observed in the areas infiltrated with YFP:28-74, rearrangement of the ER network structure was not induced (Fig. 5C, arrowheads and arrows in row 2, respectively). The perinuclear aggregates and dramatic rearrangement of the ER network structure by YFP:21-74 were similar to those observed in the YFP: nHel-induced ER membrane modification (Fig. 4B, rows 6 and 7). Thus, the ER membrane modification activity of the YFP:nHel region was retained in the YFP:21-74 mutant but was attenuated in the YFP:28-74 mutant. Consequently, comparisons between the subcellular localization of YFP:21-74 and YFP: 28-74 demonstrate that the cell death-inducing activity was correlated with the ER membrane modification exhibited by YFP: nHel and YFP:21-74.

\section{A mutation in the putative amphipathic helix of RaMV-Hel attenuates the induction of cell death and ER membrane modification.}

Because the deletion mutant analysis implied that the putative amphipathic helix is critical for the induction of cell death and membrane modification, we introduced mutations into conserved residues in the putative amphipathic helix to attempt to disrupt the helix structure. A helical wheel projection of the putative amphipathic helix-containing region (aa 36 to 71) reveals the potential to form an amphipathic helix, with hydrophobic residues including three leucines (L41, L48, and L52) on one side (Fig. 6A). These leucine residues are conserved among several comoviruses and related viruses (Fig. $8 \mathrm{C})$. Therefore, we constructed three mutants in which a single alanine residue was inserted immediately downstream of each leucine residue of the $\mathrm{nHel}$ region composed of 74 amino acids. The N-terminus of each nHel mutant was fused to YFP (YFP: L41LA, YFP:L48LA, and YFP:L52LA), and the fusion constructs were transiently overexpressed by agroinfiltration. In the areas infiltrated with YFP:L41LA, cell death was significantly compromised compared with areas infiltrated with YFP: nHel (Fig. 6B, upper left panel), and $\mathrm{H}_{2} \mathrm{O}_{2}$ production detected by $\mathrm{DAB}$ staining was also suppressed (Fig. 6B, lower left panel). In contrast, cell death and $\mathrm{H}_{2} \mathrm{O}_{2}$ production in the areas infiltrated with YFP:L48LA or YFP:L52LA not only were not compromised but occurred even more rapidly and extensively than that induced by YFP:nHel (Fig. 6B, center or right panel, respectively). These results were confirmed by ion leakage assays (Fig. 6C), which can quantify the extent of cell death. Among these three mutants in which an alanine residue was introduced next to the conserved leucine residues, only the YFP:L41LA mutant showed the cell death attenuation phenotype, but a small amount of cell death was still observed. To further 
confirm the cell death-attenuation phenotype of YFP:L41LA, we performed trypan blue staining to assess the induction of cell death and real-time RT-PCR to examine the expression levels of the defense-related genes Hinl and Hsr203j. As shown in Figure 6D, the characteristic blue coloration indicating cell death by trypan blue staining was significantly suppressed in the YFP:L41LA-infiltrated areas compared with the YFP:nHelinfiltrated areas. In addition, the expression of the defenserelated genes Hinl and Hsr203j was also suppressed in the YFP:L41LA-infiltrated areas (Fig. 6E). In contrast, cell death was not compromised but was, instead, accelerated in two of these mutants, YFP:L48LA and YFP:L52LA. Previously, Liu et al. (2009) reported that some mutations in the amphipathic helix exhibited a gain-of-function phenotype and an increase in membrane modification activity. Consistent with this report, the YFP:L48LA and YFP:L52LA mutants are believed to be gain-of-function mutants with a helix with an altered molecular structure, resulting in accelerated cell death phenotypes.

We examined the subcellular localization of all of the sitedirected mutant proteins in detail, using CLSM. To characterize the membrane modifications by these mutants, we focused on the characteristic membrane structures that are commonly induced by nHel and its deletion mutant YFP:21-27, the formation of granular structures and perinuclear aggregates and the collapse of the ER network. At 40 hpi, YFP:L41LA colocalized with the ER marker ER-CFP, whereas the formation of perinuclear aggregates derived from the ER membrane was suppressed significantly (Fig. 6F, row 1). Small granular structures closely associated with the ER network were observed in the peripheral areas of cells expressing YFP:L41LA (Fig. 6F, arrowheads in row 2). In contrast, perinuclear aggregates, rearrangement of the ER membrane, and granular structures throughout the cell were observed at 40 hpi in the YFP:L48LA- or YFP: L52LA-inoculated areas (Fig. 6F, arrows and arrowheads in rows 3 to 6), similar to the structures observed in the areas infiltrated with YFP:nHel and YFP:21-74 at 48 hpi. To measure the extent of membrane modification induced by YFP:L41LA compared with that induced by wild-type YFP:nHel, we counted the number of cells with perinuclear aggregates that occurred among randomly selected cells in the YFP:L41LA-infiltrated areas. As shown in Table 1, the percentage of cells containing perinuclear aggregates induced by expression of the YFP:L41LA mutant was much lower than in areas infiltrated with YFP:nHel. Consistent with the results of the deletion mutant analysis described above (Fig. 5), sitedirected mutational analysis of the amphipathic helix also indicated a correlation between the amphipathic helix-induced cell death and membrane modification, as manifested by the formation of perinuclear aggregates and rearrangement of the ER network.

\section{Membrane modification and cell death induction by the putative amphipathic helix require continuous lipid biosynthesis.}

As reported previously, replication of CPMV is closely associated with the intracellular membrane structure and is inhibited by cerulenin, an inhibitor of de novo membrane biosynthesis (Carette et al. 2000). This previous observation and our results

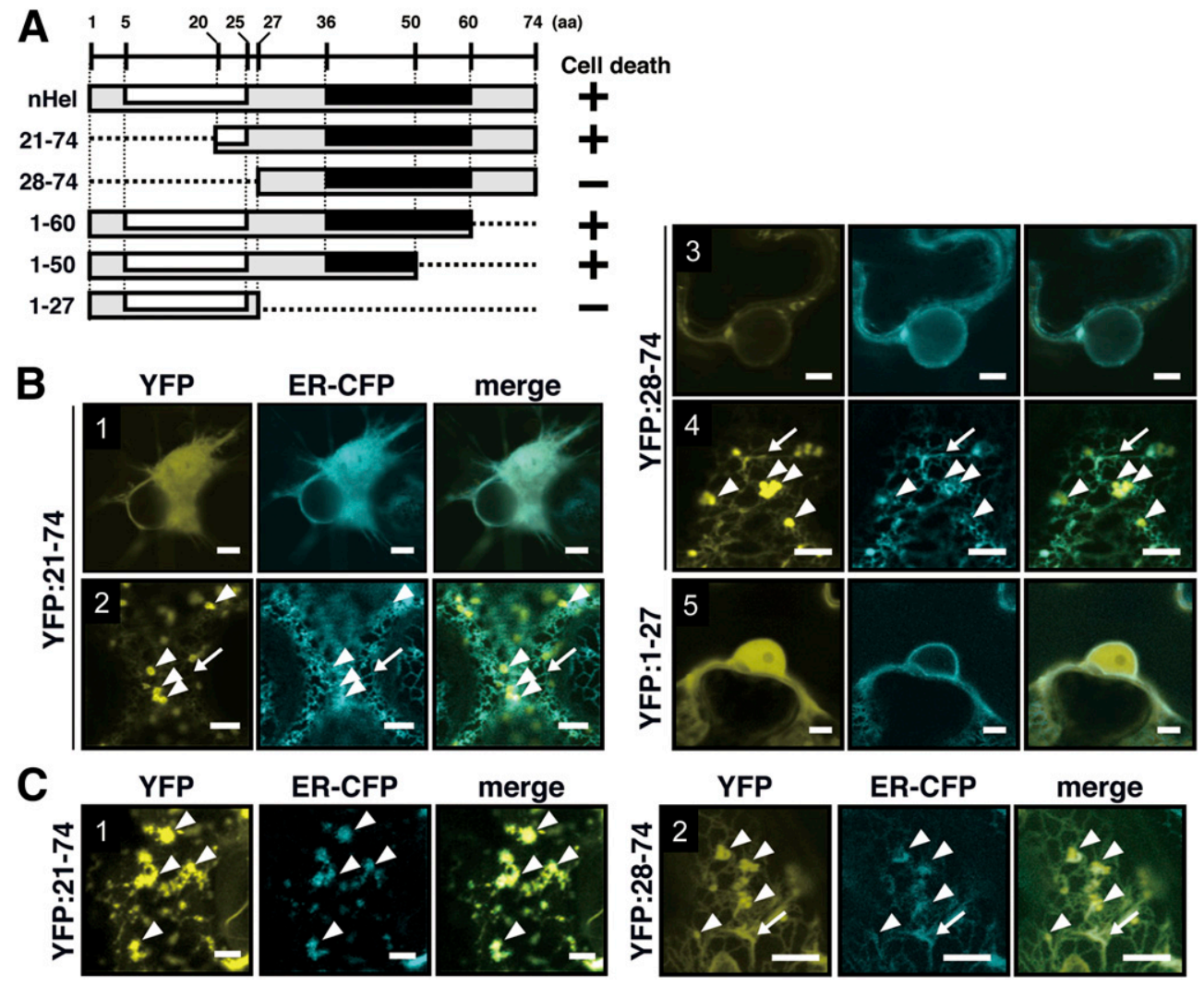

Fig. 5. Identification of the minimal N-terminal region of the Hel protein (nHel) sequences required for cell death and endoplasmic reticulum (ER) modification. A, Schematic representation of the nHel region and its deletion mutants. Full-size gray boxes correspond to the coding sequence of each construct. Numbers near the horizontal line indicate amino acid positions in the full-length Hel protein of each deletion mutant. Half-size boxes in white or black represent the putative transmembrane helix or amphipathic helix, respectively. Cell death-inducing activities of yellow fluorescent protein (YFP)-tagged deletion mutants are shown as plus or minus on the right side. YFP-tagged mutants were adjusted to an optical density at 600 nm of 0.5 . B, Subcellular localization analysis of YFP-tagged deletion mutants observed at $40 \mathrm{~h}$ postinoculation (hpi). Panels 1,3 , and 5 or 2 and 4 present close-up views of the perinuclear parts or peripheral parts of cells, respectively. C, Subcellular localization analysis of YFP:21-74 (row 1) and YFP:28-74 (row 2) observed at 48 hpi. In $\mathrm{B}$ and $\mathrm{C}$, bars in each panel represent $5 \mu \mathrm{m}$, and the left and center panels show the fluorescence of YFP-tagged constructs and ER-CFP, respectively. The right panels represent the digitally merged images and arrows and arrowheads indicate ER network structures and granular structures, respectively. 
caused us to investigate whether the membrane modification induced by the RaMV-Hel amphipathic helix was inhibited by cerulenin and whether such inhibition would compromise amphipathic helix-induced cell death.

We first examined whether RaMV accumulation was inhibited by cerulenin treatment. We inoculated RaMV using agroinfiltration of expanded $N$. benthamiana leaves in the presence or absence of $200 \mu \mathrm{M}$ cerulenin and evaluated viral accumulation at $4 \mathrm{dpi}$ in the infiltrated areas using real-time
RT-PCR. In the cerulenin-treated leaves, the accumulation of RaMV was suppressed significantly compared with the untreated control leaves (Supplementary Fig. S4). Note that the expression levels of transiently expressed Myc:GFP were not affected by cerulenin treatment at a concentration of $200 \mu \mathrm{M}$, excluding the possibility that the cerulenin treatment itself might decrease the stability of a transiently expressed protein or cellular viability. These results suggest that continuous lipid biosynthesis is required for the efficient replication of RaMV.

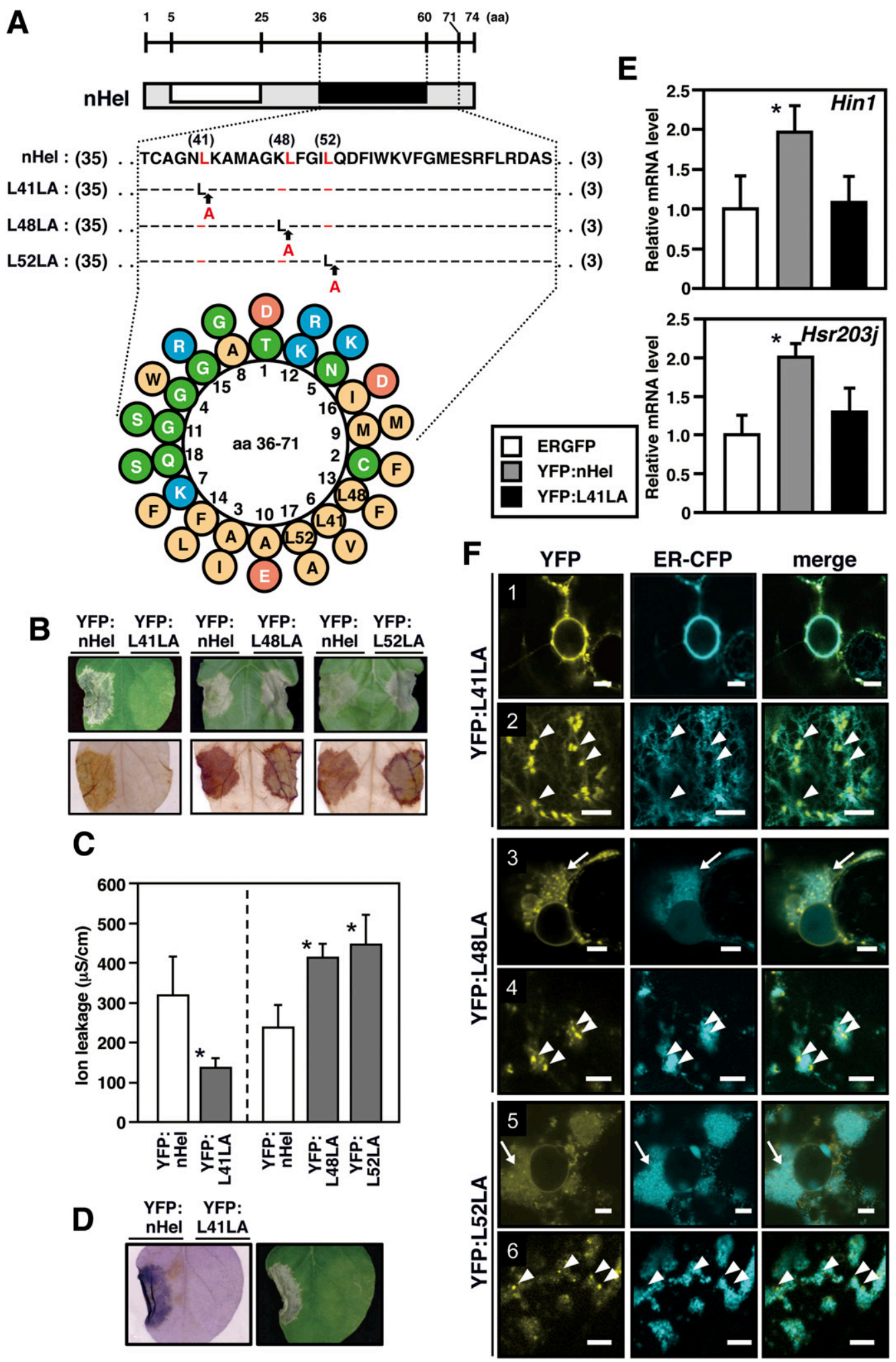


To explore whether ER membrane modification induced by the nHel region was affected by the cerulenin treatment, we coinfiltrated ER-GFP-transgenic $N$. benthamiana (16c) leaves with Myc:nHel with either $200 \mu \mathrm{M}$ cerulenin or $2 \%$ dimethyl sulfoxide (DMSO) as a control. At $24 \mathrm{~h}$ after infiltration, the plants showed perinuclear aggregates in $57.6 \%$ of the cells of the DMSO control areas (Fig. 7A, left panel; Table 2). In contrast, the membrane modification exemplified by perinuclear aggregates was observed in $17.9 \%$ of the cells of the cerulenin-treated areas (Fig. 7A, right panel; Table 2). Similar to Myc:GFP, the level of expression of YFP:nHel was not influenced significantly by cerulenin treatment (Fig. 7B). These results indicate that the membrane modification induced by the nHel region is controlled by de novo lipid biosynthesis.

We investigated whether cell death induced by the amphipathic helix was influenced by cerulenin treatment. For this purpose, we used YFP:L48LA, an nHel mutant that induces cell death more rapidly than the wild-type YFP:nHel (Fig. 6B and C). When YFP:L48LA was coinfiltrated with cerulenin, cell death and $\mathrm{H}_{2} \mathrm{O}_{2}$ production were clearly inhibited at 4 dpi compared with untreated YFP:L48LA-expressing leaves (Fig. 7C). Indeed, the level of ion leakage in the cerulenin-treated areas was significantly lower than in the control (Fig. 7D), although the expression of YFP:L48LA was not influenced by cerulenin treatment (Fig. 7E). These results suggest that inhibition of YFP:nHel-induced membrane modification by cerulenin treatment could result in the suppression of cell death. However, a small amount of cell death was still observed in the cerulenin-treated YFP:L48LA-expressing areas, indicating that cerulenin treatment may be insufficient to completely inhibit membrane synthesis. In fact, the cerulenin treatment significantly but not completely suppressed RaMV accumulation. Additionally, it remains unclear whether the inhibitory activity of cerulenin was sustained at a high level until 3 dpi under our experimental conditions.

Previous studies have shown that a mitogen-activated protein kinase (MAPK) cascade initiated by MAPKKK $\alpha$ is involved in several types of $\mathrm{R}$-mediated disease resistance. Transient overexpression of NbMAPKKK $\alpha$ activates defense signaling pathways, resulting in HR-like cell death in the absence of a pathogen (del Pozo et al. 2004). Thus, we next coinfiltrated cerulenin with Myc:NbMAPKKK $\alpha$ (Hashimoto et al. 2012) to test whether MAPKKK $\alpha$-induced cell death required lipid biosynthesis. At 3 dpi, NbMAPKKK $\alpha$-induced cell death was clearly present even in the cerulenin-treated leaves, similar to that in untreated leaves (Fig. 7F). This result was consistent with the results of ion leakage assays showing that the level of ion leakage in the cerulenin-treated
NbMAPKKK $\alpha$-expressing areas was similar to that in untreated areas (Fig. 7G). Thus, continuous lipid biosynthesis is not involved in all types of HR-like cell death, including that induced by NbMAPKKK $\alpha$.

Together, these results strengthen the notion that the amphipathic helix-induced ER membrane modification, inhibited by cerulenin treatment, was tightly correlated with its cell death-inducing activity.

\section{Overexpression of putative amphipathic helices encoded by other viruses of family Secoviridae can also induce cell death.}

Our results are suggestive of a tight correlation between the cell death-inducing activity of the amphipathic helix encoded by RaMV and its membrane modification activity. Positive-strand RNA viruses generally modify host membranes for viral replication by means of membranous proteins, such as replicases, containing transmembrane or amphipathic helices. Given the causal relationship between cell death and membrane modification, we examined whether the cell deathinducing activity of the amphipathic helix was also conserved in those of other viruses. Amphipathic helices were predicted in the N-terminal region of Hel proteins encoded by CPMV (a type species of genus Comovirus including RaMV) and Tobacco ringspot virus (TRSV; a type species of genus Nepovirus in family Secoviridae, including genus Comovirus). We constructed YFP:nHelcp and YFP:nHeltr, and YFP-tagged $\mathrm{N}$-terminal fragments of Hel proteins encoded by CPMV and TRSV, respectively, corresponding to the nHel of RaMV-Hel protein. At $3 \mathrm{dpi}$, significant cell death and $\mathrm{H}_{2} \mathrm{O}_{2}$ production were observed in the areas infiltrated with YFP:nHelcp or YFP:nHeltr (Fig. 8A). Appropriate expression of these YFPtagged fusion proteins was confirmed by Western blot analysis (Fig. 8B). However, a multiple alignment of amino acid sequences showed high variation in the $\mathrm{N}$-terminal regions of Hel proteins encoded by several secoviruses (Fig. 8C). In particular, the sequence identity of the nHel region of RaMV is $64 \%$ versus that of CPMV and showed the lowest score $(16 \%)$ with that of TRSV. Consequently, these results suggest that the cell death-inducing activity of the amphipathic helix is generally conserved among several secoviruses, despite the high level of sequence diversity among these amino acid sequences.

\section{DISCUSSION}

We investigated the molecular mechanism of necrotic symptoms in RaMV-infected $N$. benthamiana. Consistent with previous studies showing that Hel proteins encoded by two comoviruses, CPMV and BPMV, induced cell death (Carette

Fig. 6. Site-directed mutational analysis of the putative amphipathic helix in the N-terminal region of the Hel protein (nHel). A, Schematic representation of site-directed mutants of $\mathrm{nHel}$ (upper part) and a helical wheel representation of the sequence amino acids (aa) 36 to 71 in the nHel region (lower part). Sequences of the aa 36 to 71 regions of site-directed mutants are shown below the gray box. Residues identical to the nHel amino acid sequence are indicated by short horizontal lines and substituted residues are designated by red characters. Arrows indicate the positions at which alanine residues were inserted. In the lower part, each color represents nonpolar (yellow), polar or uncharged (green), acidic (pink), and basic (blue) residues. B, Symptoms elicited by yellow fluorescent protein (YFP)-tagged site-directed mutants. YFP:nHel (left) and each mutant (right) were infiltrated at an optical density at $600 \mathrm{~nm}\left(\mathrm{OD}_{600}\right)$ of 0.5 . 3,3'-Diaminobenzidine (DAB) staining was performed in the same leaves (lower panels). C, Ion leakage assays to measure cell death triggered by YFP-tagged mutants at 3 days postinoculation (dpi). Left and right parts of the graph separated by a dotted line were derived from distinct experiments. Asterisks indicate significant differences from YFP:nHel in each experiment $(P<0.05)$. Data shown represent means \pm standard deviation of five independent replicates. D, Trypan blue staining of a leaf agroinfiltrated with YFP:nHel (left) and YFP:L41LA (right) constructs at an $\mathrm{OD}_{600}$ of 0.5 (left panel). The symptoms of the same leaf are shown in the right panel. E, Expression analysis of defense-related genes in areas agroinfiltrated with a YFP:L41LA construct at an OD $_{600}$ of 0.05 . Total RNA was extracted from the infiltrated areas at 3 dpi. The values shown are relative to those in endoplasmic reticulum-green fluorescent protein (ER-GFP)-infiltrated areas. The data shown are means \pm standard deviation from four individual plants. Asterisks indicate statistically significant differences from ER-GFP-infiltrated areas $(P<0.05)$. F, Subcellular localization analysis of YFP-tagged mutants. Panels 1, 3, and 5 or 2, 4, and 6 present close-up views of the perinuclear parts or peripheral parts of cells, respectively. Bars in each panel represent $5 \mu \mathrm{m}$. Arrows and arrowheads indicate perinuclear aggregates and granular structures, respectively. 
et al. 2002; Gu and Ghabrial 2005), we showed that overexpression of RaMV-Hel can also induce cell death, directed by its N-terminal amphipathic helix. Moreover, the amphipathic helix-induced cell death has characteristics similar to HR, such as the production of $\mathrm{H}_{2} \mathrm{O}_{2}$, upregulation of defense-related genes, and SGT1 dependence (Fig. 3), as is the case with several plant-virus pathosystems (Mandadi and Scholthof 2013). Based on subcellular localization analysis, we showed that the amphipathic helix-containing region induced ER membrane modification, including the formation of perinuclear aggregates and rearrangement of the ER network, similar to the modifications observed in RaMV-infected tissues (Fig. 4). Furthermore, introduction of mutations into the amphipathic helix of Hel that disabled the cell death induction also prevented membrane modification (Figs. 5 and 6). These results indicate that the amphipathic helix-induced ER membrane proliferation is correlated with amphipathic helix-induced cell death. This was also supported by the suppression of amphipathic helixinduced membrane modification and cell death by treatment with the lipid biosynthesis inhibitor cerulenin (Fig. 7A, C, and D). However, cerulenin treatment did not compromise the HRlike cell death induced by NbMAPKKK $\alpha$, a downstream component of R-mediated disease resistance (Fig. 7F and G), suggesting that the suppression of cell death by cerulenin treatment is not common to all HR-like cell death.

Positive-strand RNA viruses have been reported to cause a variety of host membrane modifications to enable their efficient replication in infected cells. Membrane bending by amphipathic helices is assumed to be one of the major mechanisms by which membranes are modified by membrane-localized viral proteins (dos Reis Figueira et al. 2002; Rubino et al. 2007). Consistent with these findings, we found that amphipathic helices were predicted using the AmphipaSeek software in the replicase proteins of many positive-strand plant RNA viruses (Supplementary Table S2). The amphipathic helix in replicase proteins encoded by Brome mosaic virus and Tomato ringspot virus (ToRSV) is responsible for membrane rearrangement associated with virus replication (Liu et al. 2009; Zhang et al. 2005). Similar to these studies, we determined that the N-terminal amphipathic helix in the RaMV-Hel protein is also responsible for replication-associated membrane modification observed in RaMV-infected cells. We also showed that treatment with the lipid biosynthesis inhibitor cerulenin suppressed RaMV accumulation as effectively as amphipathic helix-induced membrane modification (Fig. 7), indicating that membrane modification depending on de novo lipid synthesis may facilitate virus replication. Our results are consistent with previous reports that cerulenin treatment greatly inhibits the accumulation of CPMV, Grapevine fanleaf virus, and PVX in plant protoplasts (Bamunusinghe et al. 2009; Carette et al. 2000; Ritzenthaler et al. 2002). These results indicate the important role of amphipathic helix-induced membrane modification in the replication of a wide range of plant viruses.

In this study, we demonstrated that the membrane modification activity of the amphipathic helix was tightly correlated with its cell death-inducing activity. Our results raise a new question regarding the role of the membrane structures formed due to the amphipathic helix during the induction of cell death. Based on subcellular localization analysis of the $\mathrm{nHel}$ and its mutants in Figures 4, 5 and 6, we repeatedly observed three types of distinct membrane structures, i.e., perinuclear aggregates, ER granular structures, and a collapsed ER network. These nHel-induced structures are likely to coincide with the ER membrane-derived structures formed in RaMV-infected plants (Fig. 4D). Similar perinuclear aggregates and ER granular structures have been found in CPMV-infected protoplasts (Carette et al. 2000). Viral replication factors such as CoPro and Pro-RdRp have been detected in the perinuclear region in CPMV-infected cells, suggesting that several replication proteins cooperatively form the structures important for replication at the perinuclear region (Carette et al. 2000, 2002). Therefore, the nHel-induced membrane structures are believed to be crucial for RaMV replication but are not identical to the perinuclear structures formed in RaMV-infected plants. There is no indication that these nHel-induced membrane structures have specific functions other than the replication-associated function in the induction of cell death. YFP:21-74 and YFP:L41LA, which have mutations in the nHel region, showed common changes in their membrane modification activities, suggesting that these mutations affect the conformational structures of the amphipathic helix that regulate the membrane modification activity. Because the amphipathic helix-induced cell death has characteristics similar to HR, it is possible that the amphipathic helix induces cell death mediated by R-like protein recognition, as suggested previously (Mandadi and Scholthof 2013). Alternatively, such tight correlation of cell death-inducing activity in the amphipathic helix with its membrane modification activity suggests a causal relationship between these two factors. Although the amphipathic helix encoded by the Hel protein is a common functional domain, at least in the family Secoviridae, the amino acid sequences of the RaMV nHel region and its corresponding regions of related viruses are quite diverse. In fact, the amphipathic helix of RaMV is only $16 \%$ identical to that of TRSV. It has been proposed that sequence identity in an elicitor molecule requires at least $40 \%$ in R-mediated recognition (Baurès et al. 2008). However, the amphipathic helices of CPMV and TRSV as well as that of RaMV could induce cell death. Thus, the most intriguing hypothesis is that the amphipathic helix-induced membrane modification itself or host-derived endogenous molecules released by membrane perturbation might be recognized as general elicitors, similar to damage-associated molecular patterns recognized by

Table 1. Effect of the L41LA mutation on the nHel-induced perinuclear aggregates ${ }^{\mathrm{a}}$

\begin{tabular}{|c|c|c|c|c|c|c|}
\hline Inoculum & Replicate & Positive & Negative & Total number of cells ${ }^{\mathbf{b}}$ & Chi square & $P$ value \\
\hline \multirow[t]{4}{*}{ YFP:nHel } & $\# 1$ & 41 & 1 & $42(97.6 \%)$ & & \\
\hline & \#2 & 22 & 1 & $23(95.7 \%)$ & & \\
\hline & \#3 & 52 & 1 & $53(98.1 \%)$ & & \\
\hline & total & 115 & 3 & $118(97.5 \%)$ & & \\
\hline \multirow[t]{4}{*}{ YFP:L41LA } & \#1 & 22 & 38 & $60(36.7 \%)$ & 36.3 & $1.7 \mathrm{e}-9$ \\
\hline & \#2 & 2 & 39 & $41(4.9 \%)$ & 48.0 & $4.3 \mathrm{e}-12$ \\
\hline & $\# 3$ & 14 & 41 & $55(25.5 \%)$ & 56.9 & $4.5 \mathrm{e}-14$ \\
\hline & total & 38 & 118 & $156(24.4 \%)$ & 142.6 & $2.2 \mathrm{e}-16$ \\
\hline
\end{tabular}

\footnotetext{
a The numbers of cells containing (positive) or not containing (negative) perinuclear aggregates were counted at 2 days postinoculation by observing yellow fluorescent protein (YFP) fluorescence using confocal laser scanning microscopy. This experiment was repeated three times.

b Numbers indicate the total number of cells in each experimental condition or replicate. Numbers were obtained from at least three different fields of view. The percentage of cells exhibiting perinuclear aggregates appears in parentheses. Statistical analysis was performed using Pearson's Chi-squared test with Yates' continuity correction, using R software version 3.1.2 (The R Foundation for Statistical Computing, Vienna, Austria).
} 
a host receptor protein (Boller and Felix 2009). Because the membrane modification required for virus replication is ubiquitous in a wide range of plant viruses, this hypothesis suggests a functional link between virus replication and induction of defense responses. Furthermore, such a surveillance system for monitoring host membrane structure might be a highly effective antiviral strategy of host plants.

Nevertheless, overexpression of the amphipathic helix encoded by plant viruses does not always induce cell death (Liu et al. 2009; Zhang et al. 2005). For example, the amphipathic helix in the ToRSV-encoded NTP-binding (NTB) protein does not induce cell death (Han and Sanfaçon 2003; Zhang et al. 2005). The ToRSV-NTB amphipathic helix also induces ER membrane modifications such as perinuclear aggregates and granular structures but no dramatic rearrangement of the ER
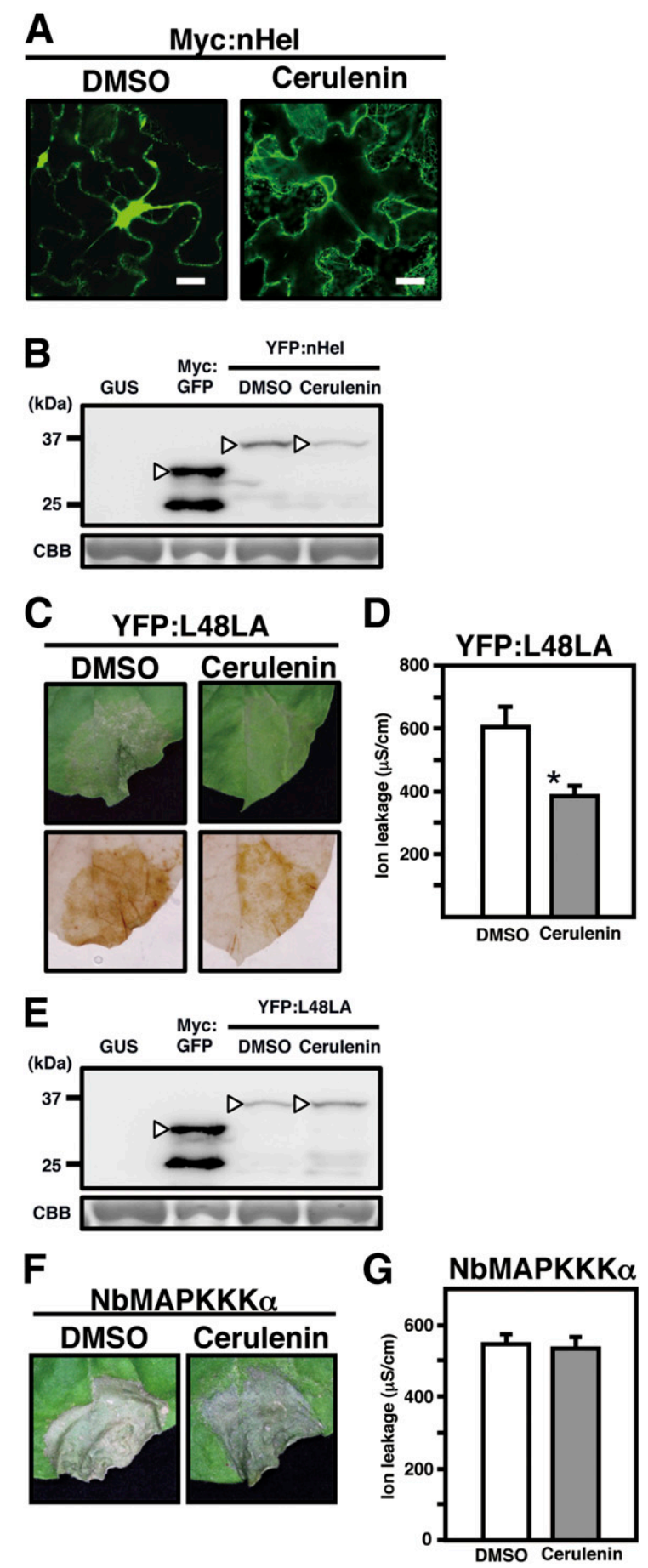

network (Han and Sanfaçon 2003; Zhang et al. 2005). The perinuclear aggregates and granular structures were similar to those induced by the amphipathic helix of RaMV-Hel. As discussed above, the differences in the membrane structures induced by $\mathrm{nHel}$ and its mutants are attributed to their membrane modification activities. Based on this hypothesis, the RaMV-Hel amphipathic helix may have higher membrane modification activity than the ToRSV-encoded NTB protein. Although any specific roles of these membrane structures in the induction of cell death remain unknown, this can explain why the ToRSV-NTB amphipathic helix does not possess cell death-inducing activity.

Our results indicate that the cell death-inducing activity of the amphipathic helix is conserved in closely related RaMV viruses. Previous studies have shown that neither CPMV nor TRSV induce systemic cell death symptoms in $N$. benthamiana (Liu and Lomonossoff 2002; Nam et al. 2011). Previously, we reported that asymptomatic virus isolates as well as symptomatic isolates encode a cell death elicitor in their replicases (Komatsu et al. 2011). This study suggests that cell death execution in virus-infected plants depends on the expression level of the elicitor, which is regulated by its replicase activity. This is consistent with the above situation in which systemic necrosis was not induced in CPMV- or TRSVinfected plants despite the cell death-inducing activity of their amphipathic helices. Together with our observation that cell death was not induced in all tissues of RaMV-infected plants, these results suggest that the amphipathic helixinduced cell death in plant cells also depends on the expression level of the amphipathic helix-including protein, interactions with other viral and host proteins, or the specific tissues in which it is expressed. This inconsistency between the symptoms in virus-infected plants and the elicitor activities of the amphipathic helices requires further studies.

\section{MATERIALS AND METHODS}

Plant material, viral isolates, and agroinfiltration.

$N$. benthamiana plants were grown in a growth chamber under $25^{\circ} \mathrm{C}$ day and $20^{\circ} \mathrm{C}$ night temperature cycles and 15-h light and 9-h dark cycles. RaMV infection was initiated using a mixture of Agrobacterium cultures carrying the binary plasmids pCAMRa1 and pCAMRa2 (Komatsu et al. 2007, 2013). All binary plasmids were transformed into Agrobacterium tumefaciens EHA105 to transiently express proteins by agroinfiltration.

\section{$\leftarrow$}

Fig. 7. Inhibitor assay on endoplasmic reticulum (ER) modification and cell death induced by the amphipathic helix. A, Effect of cerulenin treatment on Myc:nHel-induced membrane modification. Myc:nHel was infiltrated into Nicotiana benthamiana $16 \mathrm{c}$ at an $\mathrm{OD}_{600}$ of 0.5 together with $200 \mu \mathrm{M}$ cerulenin or $2 \%$ dimethyl sulfoxide (DMSO) as a control. Photos were taken at 2 days postinfiltration (dpi). Myc:nHel was infiltrated into leaves together with $200 \mu \mathrm{M}$ cerulenin or $2 \%$ dimethyl sulfoxide (DMSO) as a control. $\mathbf{B}$ and $\mathbf{E}$, Western blot analysis of yellow fluorescent protein (YFP):nHel (B) or YFP:L48LA (E) under cerulenin treatment using a monoclonal anti-GFP antibody. Arrowheads indicate specific signals of yellow fluorescent protein (YFP):nHel or Myc:GFP. $\mathbf{C}$ and $\mathbf{F}$, Symptoms of leaves inoculated with YFP:L48LA construct at an optical density at $600 \mathrm{~nm}\left(\mathrm{OD}_{600}\right)$ of $0.2(\mathrm{C}$, upper panels) or Myc:NbMAPKKK $\alpha$ construct at an $\mathrm{OD}_{600}$ of $1.0(\mathrm{~F})$ with $200 \mu \mathrm{M}$ cerulenin (right panel) or $2 \%$ DMSO (left panel) as an internal control and 3,3'-diaminobenzidine staining of the same leaves $(\mathrm{C}$, lower panels). Photos were taken at 3 or 4 days postinfiltration (dpi), respectively. $\mathbf{D}$ and $\mathbf{G}$, Ion leakage assays to measure the effect of cerulenin treatment on two types of cell death. YFP:L48LA (D) or Myc:NbMAPKKK $\alpha(\mathrm{G})$ was expressed with $200 \mu \mathrm{M}$ cerulenin (right bar) or $2 \%$ DMSO (left bar) as an internal control. Asterisks indicate significant differences from the $2 \%$ DMSO treatment in each experiment $(P<0.05)$. Data shown represent means \pm standard deviation of at least five independent replicates. 


\section{Plasmid constructs.}

The predicted RaMV-Hel coding region was PCR-amplified with KOD DNA polymerase (Toyobo, Osaka, Japan), using specific primers to construct PVX:Hel. All primers used in this study are listed in Supplementary Table S1. The resulting PCR products were inserted into the multiple cloning site of the pCAMV vector (Chapman et al. 1992; Hoshi et al. 2009). To construct transient expression vectors to express a YFP:Hel fusion protein and its derivatives, the PCR-amplified fragments were cloned into the Gateway Entry vector pENTA (Himeno et al. 2010) and were subcloned into pEarleyGate vectors (Earley et al. 2006) to create fusions with N-terminal YFP (pEarleyGate 104) or cMyc tags (pEarleyGate 203), using Gateway LR Clonase II enzyme mix (Life Technologies, Carlsbad, CA, U.S.A.). The binary vectors ER-ck or ER-gk, which express ER-localized CFP (ER-CFP) or ER-localized GFP (ER-GFP), respectively, were used to visualize the ER membrane (Nelson et al. 2007).
Real-time RT-PCR, TUNEL assay, DAB staining, silencing of $N b S G T 1$, and trypan blue staining.

All these assays followed methods described previously (Komatsu et al. 2010). In real-time RT-PCR, target transcript levels were normalized to the values of a $N$. benthamiana $18 \mathrm{~S}$ rRNA gene (Nb18S rRNA) (Hashimoto et al. 2012).

\section{Immunohistochemical analysis.}

For immunohistochemical analysis, shoot tips excised from RaMV-infected $N$. benthamiana plants were fixed and embedded in Paraplast Plus (Sherwood Medical, St. Louis, MO, U.S.A.) and were sectioned to $10 \mu \mathrm{m}$ thickness using a microtome (PR-50; Yamato Scientific, Tokyo, Japan). Sections were subsequently incubated with anti-LCP immunoglobulin G (IgG) (diluted 1:2,000) or anti-Hel IgG (diluted 1:2,000), followed by incubation with alkaline phosphatase-conjugated antirabbit IgG (Life Technologies) and visualization with nitro-blue

Table 2. Effect of cerulenin treatment on Myc:nHel-induced perinuclear aggregate formation in the endoplasmic reticulum-green fluorescent protein (ER-GFP) transgenic line Nicotiana benthamiana $16 \mathrm{c}^{\mathrm{a}}$

\begin{tabular}{|c|c|c|c|c|c|c|}
\hline Inoculum & Replicate & Positive & Negative & Total number of cells ${ }^{b}$ & Chi square & $P$ value \\
\hline \multirow[t]{4}{*}{ Myc:nHel + DMSO } & $\# 1$ & 31 & 21 & $52(59.6 \%)$ & & \\
\hline & $\# 2$ & 27 & 27 & $54(50.0 \%)$ & & \\
\hline & \#3 & 56 & 36 & $92(60.9 \%)$ & & \\
\hline & total & 114 & 84 & $198(57.6 \%)$ & & \\
\hline \multirow[t]{4}{*}{ Myc:nHel + Cerulenin } & \#1 & 6 & 45 & $51(11.8 \%)$ & 23.6 & $1.2 \mathrm{e}-6$ \\
\hline & $\# 2$ & 6 & 45 & $51(11.8 \%)$ & 16.0 & $6.1 \mathrm{e}-5$ \\
\hline & \#3 & 22 & 66 & $88(25.0 \%)$ & 22.1 & $2.6 e-6$ \\
\hline & total & 34 & 156 & $190(17.9 \%)$ & 63.0 & $2.0 \mathrm{e}-15$ \\
\hline
\end{tabular}

${ }^{a}$ Myc:nHel was coinfiltrated into $N$. benthamiana $16 \mathrm{c}$ with $200 \mu \mathrm{M}$ cerulenin or $2 \%$ dimethyl sulfoxide (DMSO) as a control. The numbers of cells containing (positive) or not containing (negative) perinuclear aggregates were counted at 24 h postinoculation by observing GFP fluorescence associated with ER-GFP using confocal laser scanning microscopy. This experiment was repeated three times.

b Numbers indicate the total number of cells in each experimental condition or replicate. Numbers of cells in each replicate were obtained from at least four different fields of view. The percentage of the cells exhibiting perinuclear aggregates appears parentheses. Statistical analysis was performed using Pearson's Chi-squared test with Yates' continuity correction, using R software version 3.1.2.

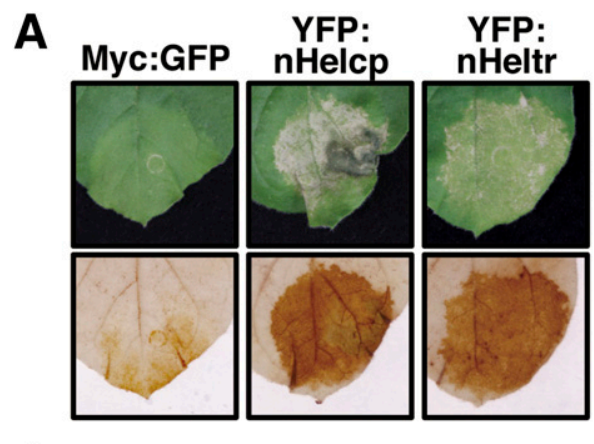

B
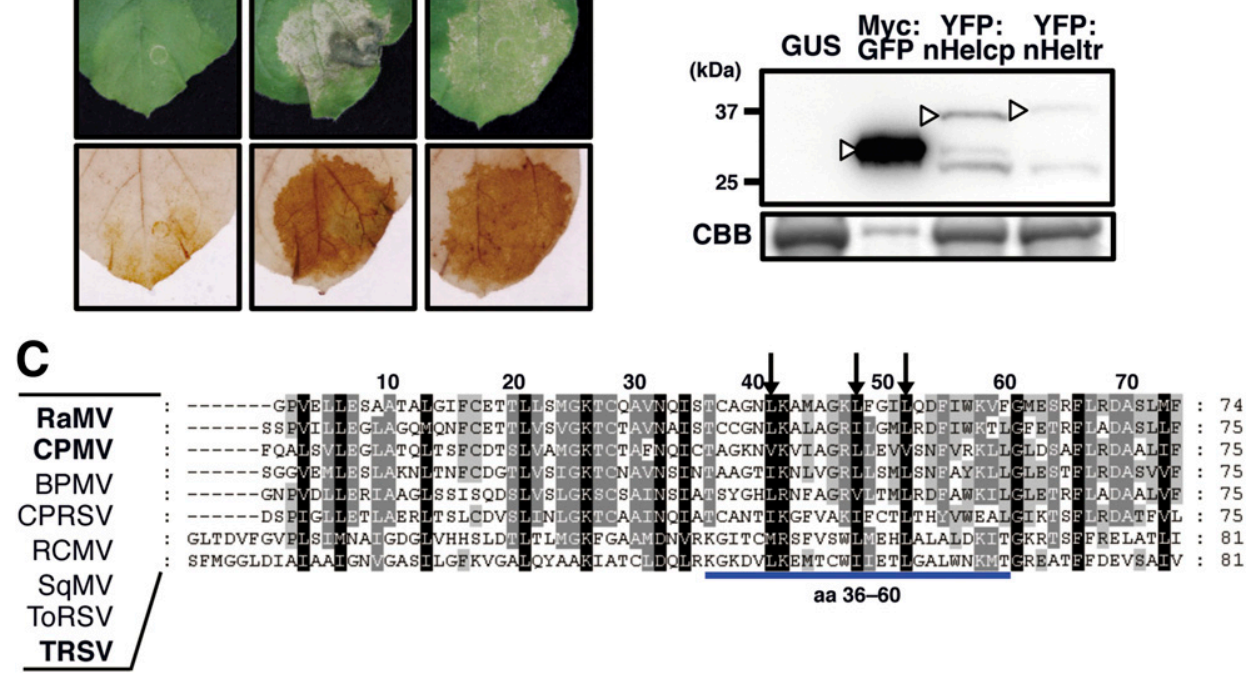

Fig. 8. Functional analysis of amphipathic helices encoded by other viruses in cell-death induction. A, Symptoms of the areas infiltrated with constructs expressing yellow fluorescent protein (YFP)-tagged amphipathic helices encoded by other viruses and 3,3'-diaminobenzidine staining of the same leaves. YFP:nHelcp and YFP:nHeltr represent the regions infected with constructs expressing portions of Cowpea mosaic virus (CPMV) or Tobacco ringspot virus (TRSV), respectively. Photos were taken at 5 days postinfiltration. B, Western blot analysis of YFP-tagged constructs using a monoclonal anti-GFP (green fluorescent protein) antibody. Arrowheads indicate specific signals of YFP-tagged proteins or Myc:GFP. C, Multiple alignment of the N-terminal regions of Hel proteins encoded by Radish mosaic virus (RaMV) and other comoviruses (CPMV, Bean pod mottle virus [BPMV], Cowpea ringspot virus [CPRSV], Red clover mottle virus [RCMV], and Squash mosaic virus [SqMV]) and nepoviruses (Tomato ringspot virus [ToRSV] and TRSV). The location of the predicted amphipathic helix of the RaMV-Hel protein (amino acids [aa] 36 to 60) and its corresponding regions in other viruses is indicated by a blue horizontal line. Arrows indicate leucine mutated residues (L41, L48, and L52). Positions are relative to each mature protein sequence. The alignment was generated using ClustalW software and presented using GeneDoc software. 
tetrazolium and 5-bromo-4-chloro-3-indolyl-phosphate. AntiLCP and anti-Hel IgG was raised as described previously (Kakizawa et al. 2001). The central region of the Hel protein corresponding to the mHel region was expressed in Escherichia coli using a pET30a vector, and the recombinant protein was used as an antigen.

\section{Bioinformatic analysis.}

Prediction of transmembrane helices or amphipathic helices in the Hel protein was performed using TMPred analysis and the SOSUI program or AmphipaSeek software and CONSENSUS secondary structure prediction method software, respectively.

\section{Microscopy.}

Fluorescence microscopy to detect the fluorescence signals produced by TUNEL assays and immunohistochemical analysis was performed using an Axio Imager microscopy (Carl Zeiss, Oberkochen, Germany).

\section{Immunoblotting.}

Proteins were separated by sodium dodecyl sulfatepolyacrylamide gel electrophoresis and were subsequently blotted onto a polyvinylidene diflouride membrane (Millipore, Billerica, MA, U.S.A.). Proteins on the membrane were detected using a monoclonal anti-GFP (Roche, Basel, Switzerland) or a monoclonal anti-c-Myc (Millipore) antibody using the chemiluminescence method (ECL-Plus immunoblotting detection system; GE Healthcare, Princeton, NJ, U.S.A.).

\section{Confocal microscopy.}

A confocal laser scanning microscope (TCS SP5; Leica Microsystems, Wetzlar, Germany) was used to visualize the subcellular distribution patterns of fluorescence proteins such as the YFP fusion proteins. The 458-, 488-, and 514-nm laser lines of an argon laser with appropriate emission filters were used to image fluorescence. GFP, YFP, or CFP fluorescence was excited at 488,514 , or $458 \mathrm{~nm}$, and emitted light was captured at 500 to $600 \mathrm{~nm}, 525$ to $600 \mathrm{~nm}$, or 465 to $505 \mathrm{~nm}$, respectively. The YFP and CFP fluorescence images were collected in a sequential manner to avoid cross talk between the channels. Images were captured digitally and were handled using the Leica LCS software.

\section{Drug treatment.}

To inhibit continuous lipid biosynthesis, $N$. benthamiana leaves were infiltrated with $200 \mu \mathrm{M}$ cerulenin (Sigma-Aldrich, St. Louis), which was dissolved in $2 \%$ DMSO-containing infiltration buffer for agroinfiltration.

\section{ACKNOWLEDGMENTS}

We thank D. Baulcombe (University of Cambridge, U.K.) for kindly providing the binary TRV and PVX constructs, the binary vector p19 constructs, and ER-localized GFP-transgenic $N$. benthamiana (16c).

\section{LITERATURE CITED}

Azevedo, C., Sadanandom, A., Kitagawa, K., Freialdenhoven, A., Shirasu, K., and Schulze-Lefert, P. 2002. The RAR1 interactor SGT1, an essential component of $R$ gene-triggered disease resistance. Science 295: 2073-2076.

Bamunusinghe, D., Hemenway, C. L., Nelson, R. S., Sanderfoot, A. A., Ye, C. M., Silva, M. A., Payton, M., and Verchot-Lubicz, J. 2009. Analysis of Potato virus $X$ replicase and TGBp3 subcellular locations. Virology 393: 272-285.

Baurès, I., Candresse, T., Leveau, A., Bendahmane, A., and Sturbois, B. 2008. The $R x$ gene confers resistance to a range of potexviruses in transgenic Nicotiana plants. Mol. Plant Microbe Interact. 21:1154-1164.
Boller, T., and Felix, G. 2009. A renaissance of elicitors: Perception of microbe-associated molecular patterns and danger signals by patternrecognition receptors. Annu. Rev. Plant Biol. 60:379-406.

Carette, J. E., Stuiver, M., Van Lent, J., Wellink, J., and Van Kammen, A 2000. Cowpea mosaic virus infection induces a massive proliferation of endoplasmic reticulum but not Golgi membranes and is dependent on de novo membrane synthesis. J. Virol. 74:6556-6563.

Carette, J. E., van Lent, J., MacFarlane, S. A., Wellink, J., and van Kammen, A. 2002. Cowpea mosaic virus 32- and 60-kilodalton replication proteins target and change the morphology of endoplasmic reticulum membranes. J. Virol. 76:6293-6301.

Chapman, S., Kavanagh, T., and Baulcombe, D. 1992. Potato virus $X$ as a vector for gene expression in plants. Plant J. 2:549-557.

del Pozo, O., Pedley, K. F., and Martin, G. B. 2004. MAPKKK $\alpha$ is a positive regulator of cell death associated with both plant immunity and disease. EMBO (Eur. Mol. Biol. Organ.) J. 23:3072-3082.

den Boon, J. A., and Ahlquist, P. 2010. Organelle-like membrane compartmentalization of positive-strand RNA virus replication factories. Annu. Rev. Microbiol. 64:241-256.

den Boon, J. A., Chen, J., and Ahlquist, P. 2001. Identification of sequences in Brome mosaic virus replicase protein 1a that mediate association with endoplasmic reticulum membranes. J. Virol. 75:12370-12381.

Diaz, A., Wang, X., and Ahlquist, P. 2010. Membrane-shaping host reticulon proteins play crucial roles in viral RNA replication compartment formation and function. Proc. Natl. Acad. Sci. U.S.A. 107:16291-16296.

Dinesh-Kumar, S. P., Tham, W. H., and Baker, B. J. 2000. Structurefunction analysis of the Tobacco mosaic virus resistance gene $N$. Proc. Natl. Acad. Sci. U.S.A. 97:14789-14794.

dos Reis Figueira, A., Golem, S., Goregaoker, S. P., and Culver, J. N. 2002. A nuclear localization signal and a membrane association domain contribute to the cellular localization of the Tobacco mosaic virus $126-\mathrm{kDa}$ replicase protein. Virology 301:81-89.

Drin, G., and Antonny, B. 2010. Amphipathic helices and membrane curvature. FEBS Lett. 584:1840-1847.

Earley, K. W., Haag, J. R., Pontes, O., Opper, K., Juehne, T., Song, K., and Pikaard, C. S. 2006. Gateway-compatible vectors for plant functional genomics and proteomics. Plant J. 45:616-629.

Goodin, M., Yelton, S., Ghosh, D., Mathews, S., and Lesnaw, J. 2005. Livecell imaging of rhabdovirus-induced morphological changes in plan nuclear membranes. Mol. Plant Microbe Interact. 18:703-709.

Gouttenoire, J., Penin, F., and Moradpour, D. 2010. Hepatitis C virus nonstructural protein 4B: A journey into unexplored territory. Rev. Med. Virol. 20:117-129.

Gu, H., and Ghabrial, S. A. 2005. The Bean pod mottle virus proteinase cofactor and putative helicase are symptom severity determinants. Virology 33:271-283.

Han, S., and Sanfaçon, H. 2003. Tomato ringspot virus proteins containing the nucleoside triphosphate binding domain are transmembrane proteins that associate with the endoplasmic reticulum and cofractionate with replication complexes. J. Virol. 77:523-534.

Hashimoto, M., Komatsu, K., Maejima, K., Okano, Y., Shiraishi, T., Ishikawa, K., Takinami, Y., Yamaji, Y., and Namba, S. 2012. Identification of three MAPKKKs forming a linear signaling pathway leading to programmed cell death in Nicotiana benthamiana. BMC Plant Biol. 12:103.

Himeno, M., Maejima, K., Komatsu, K., Ozeki, J., Hashimoto, M., Kagiwada, S., Yamaji, Y., and Namba, S. 2010. Significantly low level of small RNA accumulation derived from an encapsidated mycovirus with dsRNA genome. Virology 396:69-75.

Horner, S. M., Park, H. S., and Gale, M., Jr. 2012. Control of innate immune signaling and membrane targeting by the Hepatitis $C$ virus NS3/4A protease are governed by the NS3 helix $\alpha_{0}$. J. Virol. 86:3112-3120.

Hoshi, A., Oshima, K., Kakizawa, S., Ishii, Y., Ozeki, J., Hashimoto, M., Komatsu, K., Kagiwada, S., Yamaji, Y., and Namba, S. 2009. A unique virulence factor for proliferation and dwarfism in plants identified from a phytopathogenic bacterium. Proc. Natl. Acad. Sci. U.S.A. 106:6416-6421.

Kakizawa, S., Oshima, K., Kuboyama, T., Nishigawa, H., Jung, H., Sawayanagi, T., Tsuchizaki, T., Miyata, S., Ugaki, M., and Namba, S. 2001. Cloning and expression analysis of phytoplasma protein translocation genes. Mol. Plant Microbe Interact. 14:1043-1050.

Komatsu, K., Hashimoto, M., Maejima, K., Ozeki, J., Kagiwada, S., Takahashi, S., Yamaji, Y., and Namba, S. 2007. Genome sequence of a Japanese isolate of Radish mosaic virus: The first complete nucleotide sequence of a crucifer-infecting comovirus. Arch. Virol. 152:1501-1506.

Komatsu, K., Hashimoto, M., Ozeki, J., Yamaji, Y., Maejima, K., Senshu, H., Himeno, M., Okano, Y., Kagiwada, S., and Namba, S. 2010. Viralinduced systemic necrosis in plants involves both programmed cell death and the inhibition of viral multiplication, which are regulated by independent pathways. Mol. Plant Microbe Interact. 23:283-293. 
Komatsu, K., Hashimoto, M., Maejima, K., Shiraishi, T., Neriya, Y., Miura, C., Minato, N., Okano, Y., Sugawara, K., Yamaji, Y., and Namba, S. 2011. A necrosis-inducing elicitor domain encoded by both symptomatic and asymptomatic Plantago asiatica mosaic virus isolates, whose expression is modulated by virus replication. Mol. Plant-Microbe Interact. 24: 408-420.

Komatsu, K., Hashimoto, M., Okano, Y., Keima, T., Kitazawa, Y., Nijo, T., Takahashi, S., Maejima, K., Yamaji, Y., and Namba, S. 2013. Construction of an infectious cDNA clone of Radish mosaic virus, a crucifer-infecting comovirus. Arch. Virol. 158:1579-1582.

Laliberté, J. F., and Sanfaçon, H. 2010. Cellular remodeling during plant virus infection. Annu. Rev. Phytopathol. 48:69-91.

Liu, L., and Lomonossoff, G. 2002. Agroinfection as a rapid method for propagating Cowpea mosaic virus-based constructs. J. Virol. Methods 105:343-348.

Liu, L., Westler, W. M., den Boon, J. A., Wang, X., Diaz, A., Steinberg, H. A., and Ahlquist, P. 2009. An amphipathic $\alpha$-helix controls multiple roles of Brome mosaic virus protein 1a in RNA replication complex assembly and function. PLoS Pathog. 5:e1000351.

Mandadi, K. K., and Scholthof, K. B. 2013. Plant immune responses against viruses: How does a virus cause disease? Plant Cell 25:1489-1505.

Miller, S., and Krijnse-Locker, J. 2008. Modification of intracellular membrane structures for virus replication. Nat. Rev. Microbiol. 6:363-374.

Moffett, P. 2009. Mechanisms of recognition in dominant $R$ gene mediated resistance. Adv. Virus Res. 75:1-33.

Nam, M., Koh, S., Kim, S. U., Domier, L. L., Jeon, J. H., Kim, H. G., Lee, S.-H., Bent, A. F., and Moon, J. S. 2011. Arabidopsis TTRl causes LRRdependent lethal systemic necrosis, rather than systemic acquired resistance, to Tobacco ringspot virus. Mol. Cells 32:421-429.

Nelson, B. K., Cai, X., and Nebenführ, A. 2007. A multicolored set of in vivo organelle markers for co-localization studies in Arabidopsis and other plants. Plant J. 51:1126-1136.

Peart, J. R., Lu, R., Sadanandom, A., Malcuit, I., Moffett, P., Brice, D. C., Schauser, L., Jaggard, D. A., Xiao, S., Coleman, M. J., Dow, M., Jones, J. D., Shirasu, K., and Baulcombe, D. C. 2002. Ubiquitin ligaseassociated protein SGT1 is required for host and nonhost disease resistance in plants. Proc. Natl. Acad. Sci. U.S.A. 99:10865-10869.

Pruss, G. J., Nester, E. W., and Vance, V. 2008. Infiltration with Agrobacterium tumefaciens induces host defense and development-dependent responses in the infiltrated zone. Mol. Plant Microbe Interact. 21:1528-1538.

Ratcliff, F., Martin-Hernandez, A. M., and Baulcombe, D. C. 2001. Technical Advance. Tobacco rattle virus as a vector for analysis of gene function by silencing. Plant J. 25:237-245.

Ritzenthaler, C., Laporte, C., Gaire, F., Dunoyer, P., Schmitt, C., Duval, S., Piéquet, A., Loudes, A. M., Rohfritsch, O., Stussi-Garaud, C., and Pfeiffer, P. 2002. Grapevine fanleaf virus replication occurs on endoplasmic reticulum-derived membranes. J. Virol. 76:8808-8819.

Rubino, L., Navarro, B., and Russo, M. 2007. Cymbidium ringspot virus defective interfering RNA replication in yeast cells occurs on endoplasmic reticulum-derived membranes in the absence of peroxisomes. J. Gen. Virol. 88:1634-1642.

Ruiz, M. T., Voinnet, O., and Baulcombe, D. C. 1998. Initiation and maintenance of virus-induced gene silencing. Plant Cell 10:937-946.

Sanfaçon, H. 2012. Investigating the role of viral integral membrane proteins in promoting the assembly of nepovirus and comovirus replication factories. Front. Plant Sci. 3:313.

Sanfaçon, H., Wellink, J., Le Gall, O., Karasev, A., van der Vlugt, R., and Wetzel, T. 2009. Secoviridae: A proposed family of plant viruses within the order Picornavirales that combines the families Sequiviridae and Comoviridae, the unassigned genera Cheravirus and Sadwavirus, and the proposed genus Torradovirus. Arch. Virol. 154:899-907.

Schwartz, M., Chen, J., Janda, M., Sullivan, M., den Boon, J., and Ahlquist, P. 2002. A positive-strand RNA virus replication complex parallels form and function of retrovirus capsids. Mol. Cell 9:505-514.

Sekine, K. T., Ishihara, T., Hase, S., Kusano, T., Shah, J., and Takahashi, H. 2006. Single amino acid alterations in Arabidopsis thaliana RCY1 compromise resistance to Cucumber mosaic virus, but differentially suppress hypersensitive response-like cell death. Plant Mol. Biol. 62:669-682.

Seo, Y. S., Rojas, M. R., Lee, J. Y., Lee, S. W., Jeon, J. S., Ronald, P., Lucas, W. J., and Gilbertson, R. L. 2006. A viral resistance gene from common bean functions across plant families and is up-regulated in a non-virusspecific manner. Proc. Natl. Acad. Sci. U.S.A. 103:11856-11861.

Turner, K. A., Sit, T. L., Callaway, A. S., Allen, N. S., and Lommel, S. A. 2004. Red clover necrotic mosaic virus replication proteins accumulate at the endoplasmic reticulum. Virology 320:276-290.

Xu, P., and Roossinck, M. J. 2000. Cucumber mosaic virus D satellite RNAinduced programmed cell death in tomato. Plant Cell 12:1079-1092.

$\mathrm{Xu}$, P., Blancaflor, E. B., and Roossinck, M. J. 2003. In spite of induced multiple defense responses, tomato plants infected with Cucumber mosaic virus and D satellite RNA succumb to systemic necrosis. Mol. Plant Microbe Interact. 16:467-476.

Zhang, S. C., Zhang, G., Yang, L., Chisholm, J., and Sanfaçon, H. 2005. Evidence that insertion of Tomato ringspot nepovirus NTB-VPg protein in endoplasmic reticulum membranes is directed by two domains: A $\mathrm{C}$-terminal transmembrane helix and an $\mathrm{N}$-terminal amphipathic helix. J. Virol. 79:11752-11765.

\section{AUTHOR-RECOMMENDED INTERNET RESOURCES}

AmphipaSeek (amphipathic in-plane membrane anchors prediction) software: https://npsa-pbil.ibcp.fr/cgi-bin/npsa_automat.pl?page=/NPSA/ npsa_amphipaseek.html

Consensus Secondary Structure Prediction software:

http://www.bioinf.manchester.ac.uk/dbbrowser/bioactivity/NPS2.html

SOSUI program: http://bp.nuap.nagoya-u.ac.jp/sosui

The TMPred prediction program:

http://www.ch.embnet.org/software/TMPRED_form.html 